TRANSACTIONS OF THE

AMERICAN MATHEMATICAL SOCIETY

Volume 363, Number 9, September 2011, Pages 4801-4829

S 0002-9947(2011)05301-8

Article electronically published on March 31, 2011

\title{
MONODROMY CONJECTURE FOR NONDEGENERATE SURFACE SINGULARITIES
}

\author{
ANN LEMAHIEU AND LISE VAN PROEYEN
}

\begin{abstract}
We prove the monodromy conjecture for the topological zeta function for all nondegenerate surface singularities. Fundamental in our work is a detailed study of the formula for the zeta function of monodromy by Varchenko and the study of the candidate poles of the topological zeta function yielded by what we call ' $B_{1}$-facets'. In particular, new cases among the nondegenerate surface singularities for which the monodromy conjecture is now proven are the nonisolated singularities, the singularities giving rise to a topological zeta function with multiple candidate poles and the ones for which the Newton polyhedron contains a $B_{1}$-facet.
\end{abstract}

\section{INTRODUCTION}

In We65, Weil introduced some zeta functions $\mathcal{Z}(K, f)$ that are integrals over a $p$-adic field $K$ and that are associated to a polynomial $f(\underline{x}) \in K[\underline{x}]$. Igusa showed that these zeta functions are rational and he studied their poles (see Ig75 and Ig78). The analogous integrals over $K=\mathbb{R}$ or $\mathbb{C}$ are meromorphic (see for example [At70 and BeGe69), and it is known that their poles are contained in the set of roots, and roots shifted by a negative integer, of the Bernstein polynomial $b_{f}$. By Malgrange (Ma83), if $\alpha$ is a root of $b_{f}$, then $e^{2 \pi i \alpha}$ is an eigenvalue of the local monodromy of $f$ at some point of $f^{-1}(0)$. So when $K=\mathbb{R}$ or $\mathbb{C}$, the poles of the zeta function induce eigenvalues of the local monodromy. The study of concrete examples made it natural to propose the following two conjectures at the $p$-adic side.

Monodromy conjecture ([Ig88). Let $F \subset \mathbb{C}$ be a number field and $f \in F[\underline{x}]$. For almost all $p$-adic completions $K$ of $F$, if $s_{0}$ is a pole of $\mathcal{Z}(K, f)$, then $e^{2 \pi i R e\left(s_{0}\right)}$ is an eigenvalue of the local monodromy of $f$ at some point of the hypersurface $f=0$.

The stronger conjecture at the level of the Bernstein-Sato polynomial is ([Ig88]): Let $F \subset \mathbb{C}$ be a number field and $f \in F[\underline{x}]$. For almost all $p$-adic completions $K$ of $F$, if $s_{0}$ is a pole of $\mathcal{Z}(K, f)$, then $s_{0}$ is a root of the (local) Bernstein-Sato polynomial $b_{f, 0}(s)$ of $f$.

One calls this zeta function $\mathcal{Z}(K, f)$ the Igusa zeta function. When Denef and Loeser introduced the topological zeta function in 1992 as a kind of limit of the Igusa zeta function (DL92]), an analogous version of the monodromy conjecture arose.

Received by the editors November 9, 2009.

2010 Mathematics Subject Classification. Primary 14B05.

The first author's research was partially supported by the Fund of Scientific Research - Flanders and MEC PN I+D+I MTM2007-64704. (C) 2011 American Mathematical Society
Reverts to public domain 28 years from publication 
The monodromy conjecture for the Igusa zeta function implies the monodromy conjecture for the topological zeta function.

The conjecture has been proven in several cases. Loeser verified the conjecture for the Igusa zeta function at the level of the Bernstein polynomial for plane curves (see [L88]). He also gave a proof for a class of polynomials in higher dimensions; the polynomial should be nondegenerate with respect to its Newton polyhedron and should satisfy some numerical conditions ([L90] and Section 2). Rodrigues gave an alternative proof for the monodromy conjecture for plane curves (see Ro04). Artal Bartolo, Cassou-Noguès, Luengo and Melle Hernández proved the monodromy conjecture for some surface singularities, such as for the topological zeta function for the superisolated ones (see $\mathrm{A}-\mathrm{B}, \mathrm{C}-\mathrm{N}]$ ), and for the Igusa zeta function for the quasiordinary polynomials in A-B,C-N1. In LV09, Veys and the first author proved the monodromy conjecture for the topological zeta function for surface singularities that are general for a toric idealistic cluster.

In this paper we prove the monodromy conjecture for the topological zeta function for all nondegenerate surface singularities. In Section 1 we explain the objects that play a role in the monodromy conjecture, in particular the zeta function of monodromy and the local topological zeta function. When the singularity is nondegenerate with respect to its Newton polyhedron, then these zeta functions can be expressed in terms of their Newton polyhedron. We recall these formulas and some basic notions in the context of nondegenerate singularities in Section 2. Here we also make precise the conditions that the singularities have to satisfy to be covered by the proof of [L90] and which we can omit in this paper in dimension 3. In Section 3 we explain the strategy behind our proof and execute it. Fundamental is an analysis of the formula of Varchenko for the zeta function of monodromy ( $\operatorname{Var} 76]$ ). This allows us to show in dimensions 2 and 3 that all facets induce eigenvalues of monodromy, except some special facets that we call $B_{1}$-facets. In Section 4 we will determine when these $B_{1}$-facets contribute a pole of the local topological zeta function. Mostly they do not. When they do contribute a pole, then we determine a vertex of the Newton polyhedron that gives rise to the corresponding eigenvalue of monodromy. A combination of these results leads to the monodromy conjecture for nondegenerate polynomials in dimensions 2 and 3. Although already completely known in dimension 2 (see L88), we still mention here our results in dimension 2 because we need them in dimension 3. In Section 5 we provide some examples.

At first glance it seems that the same strategy could lead to a proof in arbitrary dimensions, but the combinatorics are getting much more tricky and probably some new tools are needed.

\section{The MONODROMY CONJECTURE}

Let $f:\left(\mathbb{C}^{n}, 0\right) \rightarrow(\mathbb{C}, 0)$ be a germ of a holomorphic function. Let $F$ be the Milnor fibre of the Milnor fibration at the origin associated with $f$ and write $h^{*}$ : $H^{i}(F, \mathbb{C}) \rightarrow H^{i}(F, \mathbb{C}), i \geq 0$, for the monodromy transformation.

Definition 1. The zeta function of monodromy $Z(f, 0)$ at the origin associated to the polynomial $f$ is

$$
Z(f, 0)(t):=\prod_{i \geq 0}\left(\operatorname{det}\left(i d^{*}-t h^{*} ; H^{i}(F, \mathbb{C})\right)\right)^{(-1)^{(i+1)}} .
$$


One calls $\alpha$ an eigenvalue of monodromy of $f$ at the origin if $\alpha$ is an eigenvalue for some $h^{*}: H^{i}(F, \mathbb{C}) \rightarrow H^{i}(F, \mathbb{C})$.

In 1992, Denef and Loeser created a new zeta function which they called the topological zeta function because of the topological Euler-Poincaré characteristic $\chi(\cdot)$ turning up in it. Let $\pi: Z \rightarrow \mathbb{C}^{n}$ be an embedded resolution of singularities of $f^{-1}\{0\}$. We write $E_{j}, j \in S$, for the irreducible components of $\pi^{-1}\left(f^{-1}\{0\}\right)$ and we denote by $N_{j}$ and by $\nu_{j}-1$ the multiplicities of $E_{j}$ in the divisor on $Z$ of $f \circ \pi$ and $\pi^{*}\left(d x_{1} \wedge \ldots \wedge d x_{n}\right)$, respectively. We also denote $E_{I}:=\bigcap_{i \in I} E_{i}$ and $E_{I}^{\circ}:=E_{I} \backslash\left(\bigcup_{j \notin I} E_{j}\right)$, for $I \subset S$. Let the $E_{j}, j \in J:=\{1, \ldots, r\} \subset S$, be the exceptional irreducible components of $\pi^{-1}(\{0\})$.

Definition 2. The local topological zeta function at the origin associated to $f$ is the rational function in one complex variable

$$
Z_{t o p, f}(s):=\sum_{I \subset S} \chi\left(E_{I}^{\circ} \cap \pi^{-1}\{0\}\right) \prod_{i \in I} \frac{1}{N_{i} s+\nu_{i}} .
$$

Denef and Loeser proved in DL92 that every embedded resolution gives rise to the same function, so the topological zeta function is a well-defined singularity invariant. They also formulated the analogous version of the monodromy conjecture for the local topological zeta function.

Monodromy conjecture for the topological zeta function. If $s_{0}$ is a pole of $Z_{t o p, f}$, then $e^{2 \pi i s_{0}}$ is an eigenvalue of monodromy of $f$ at some point of the germ at 0 of the hypersurface $f=0$.

\section{Nondegenerate Singularities}

Let $f \in \mathbb{C}\left[x_{1}, \ldots, x_{n}\right]$ be a nonconstant polynomial satisfying $f(0)=0$. We write $f=\sum_{k \in \mathbb{Z}_{\geq 0}^{n}} a_{k} x^{k}$, where $k=\left(k_{1}, \ldots, k_{n}\right)$ and $x^{k}=x_{1}^{k_{1}} \cdot \ldots \cdot x_{n}^{k_{n}}$. The support of $f$ is supp $f=\left\{k \in \mathbb{Z}_{\geq 0}^{n} \mid a_{k} \neq 0\right\}$. The Newton polyhedron $\Gamma_{0}$ of $f$ at the origin is the convex hull in $\mathbb{R}_{\geq 0}^{n}$ of

$$
\bigcup_{k \in \operatorname{supp} f} k+\mathbb{R}_{\geq 0}^{n} .
$$

A face of the Newton polyhedron is the intersection of $\Gamma_{0}$ with a supporting hyperplane. A facet is a face of dimension $n-1$. A polynomial $f\left(x_{1}, \ldots, x_{n}\right)$ is called nondegenerate with respect to its Newton polyhedron $\Gamma_{0}$ if for every compact face $\tau$ of $\Gamma_{0}$ the polynomials $f_{\tau}:=\sum_{k \in \tau} a_{k} x^{k}$ and $\partial f_{\tau} / \partial x_{i}, 1 \leq i \leq n$, have no common zeroes in $(\mathbb{C} \backslash\{0\})^{n}$. For a fixed Newton polyhedron $\Gamma$, almost all polynomials having $\Gamma$ as its Newton polyhedron are nondegenerate with respect to $\Gamma$ (see AVG86, p.157]).

For nondegenerate polynomials many invariants can be deduced from the Newton polyhedron. For instance it is possible to express the local topological zeta function and the monodromy zeta function in terms of the Newton polyhedron. Before giving these formulas, we recall the needed notions. If $a_{1}, \ldots, a_{r} \in \mathbb{R}^{n} \backslash\{0\}$, we call cone $\left(a_{1}, \ldots, a_{r}\right)=\left\{\sum_{i=1}^{r} \lambda_{i} a_{i} \mid \lambda_{i} \in \mathbb{R}, \lambda_{i}>0\right\}$ the cone strictly positively spanned by the vectors $a_{1}, \ldots, a_{r}$. Fix a cone $\Delta$. If there exist vectors $a_{1}, \ldots, a_{r} \in \mathbb{R}^{n} \backslash\{0\}$ that are linearly independent over $\mathbb{R}$ such that $\Delta=\operatorname{cone}\left(a_{1}, \ldots, a_{r}\right)$, then $\Delta$ is called a simplicial cone. If moreover $a_{1}, \ldots, a_{r}$ can be chosen in $\mathbb{Z}^{n}$, we say $\Delta$ is a rational simplicial cone. For a rational simplicial cone $\Delta$ spanned by the primitive 
and linearly independent vectors $a_{1}, \ldots, a_{r} \in \mathbb{Z}^{n}$ we define the multiplicity of $\Delta$, denoted by mult $(\Delta)$, as the index of the lattice $\mathbb{Z} a_{1}+\cdots+\mathbb{Z} a_{r}$ in the group of the points with integral coordinates of the vector space generated by $a_{1}, \ldots, a_{r}$. It is easy to verify that the multiplicity of $\Delta$ equals the number of elements in the set

$$
\mathbb{Z}^{n} \cap\left\{\sum_{i=1}^{r} \lambda_{i} a_{i} \mid 0 \leq \lambda_{i}<1 \text { for } i=1, \ldots, r\right\} .
$$

Moreover, $\operatorname{mult}(\Delta)$ is equal to the greatest common divisor of the determinants of the $(r \times r)$-matrices obtained by omitting columns from the matrix $A$ with rows $a_{1}, \ldots, a_{r}$.

Let $\Gamma_{0}$ be a Newton polyhedron in $\mathbb{R}^{n}$. For $a=\left(a_{1}, \ldots, a_{n}\right) \in \mathbb{R}_{\geq 0}^{n}$ we put $N(a):=\inf _{x \in \Gamma_{0}} a \cdot x, \nu(a):=\sum_{i=1}^{n} a_{i}$ and $F(a):=\left\{x \in \Gamma_{0} \mid a \cdot x=\bar{N}(a)\right\}$. All $F(a), a \neq 0$, are faces of $\Gamma_{0}$. To a face $\tau$ of $\Gamma_{0}$ one associates a dual cone $\tau^{\circ} \subset \mathbb{R}^{n}$, defined as the closure in $\mathbb{R}^{n}$ of $\left\{a \in \mathbb{R}_{\geq 0}^{n} \mid F(a)=\tau\right\}$. This is a rational convex cone with vertex the origin of dimension $n-\operatorname{dim} \tau$. Note that the map $\tau \mapsto \tau^{\circ}$ is inclusion-reversing. In particular if $\operatorname{dim} \tau=n-1$, then $\tau^{\circ}$ is a ray, say $\tau^{\circ}=a \mathbb{R}_{\geq 0}$ for some $a \in \mathbb{Z}_{>0}^{n}$, and then the equation of the hyperplane through $\tau$ is $a \cdot x=N(\bar{a})$. If we demand that $\operatorname{gcd}\left(a_{1}, \ldots, a_{n}\right)=1$, this $a$ is uniquely defined. For a facet $\tau$ we also use the notation $N(\tau)$ and $\nu(\tau)$, meaning respectively $N(a)$ and $\nu(a)$ for this associated $a \in \mathbb{Z}_{>0}^{n}$.

Suppose $\Delta=\overline{\mathbb{R}}_{\geq 0} a_{1}+\cdots+\mathbb{R}_{\geq 0} a_{r}$, with $a_{1}, \ldots, a_{r} \in \mathbb{Z}_{\geq 0}^{n}$ linearly independent and primitive (i.e. with relatively prime components). Then we put

$$
J_{\Delta}(s):=\frac{\operatorname{mult}(\Delta)}{\prod_{i=1}^{r}\left(N\left(a_{i}\right) s+\nu\left(a_{i}\right)\right)} .
$$

The dual cone $\tau^{\circ}$ is not necessarily a rational simplicial cone. However, there always exists a decomposition $\tau^{\circ}=\bigcup_{i=1}^{k} \Delta_{i}$ of $\tau^{\circ}$ in rational simplicial cones $\Delta_{i}$ of dimension $\ell=\operatorname{dim} \tau^{\circ}$ satisfying $\operatorname{dim}\left(\Delta_{i} \cap \Delta_{j}\right)<\ell$ if $i \neq j$. Such a decomposition is even possible without introducing new rays (this is well known; for a proof see D95. Lemma 2]). To an arbitrary face $\tau$ of $\Gamma_{0}$ we now associate the rational function

$$
J_{\tau}(s):=\sum_{i=1}^{k} J_{\Delta_{i}}(s)
$$

where $\tau^{\circ}=\bigcup_{i=1}^{k} \Delta_{i}$ is a decomposition as mentioned above. Denef and Loeser showed in [DL92, Lemme 5.1.1] that this function does not depend on the chosen decomposition of $\tau^{\circ}$.

Theorem 1 ([DL92, Théorème 5.3]). If $f$ is nondegenerate with respect to $\Gamma_{0}$, then the local topological zeta function at the origin is equal to

$$
Z_{\text {top }, f}(s)=\sum_{\tau \text { vertex of } \Gamma_{0}} J_{\tau}(s)+\frac{s}{s+1} \sum_{\begin{array}{c}
\tau \text { compact } \\
\text { face of } \Gamma_{0}, \\
\operatorname{dim} \tau \geq 1
\end{array}}(-1)^{\operatorname{dim} \tau}(\operatorname{dim} \tau) ! \operatorname{Vol}(\tau) J_{\tau}(s)
$$

For a face $\tau$ of dimension 0 , we $\operatorname{put} \operatorname{Vol}(\tau)=1$. For every other compact face, $\operatorname{Vol}(\tau)$ is defined as the volume of $\tau$ for the volume form $\omega_{\tau}$. This is a volume form on $\operatorname{Aff}(\tau)$, the affine space spanned by $\tau$, such that the parallelepiped spanned by a lattice-basis of $\mathbb{Z}^{n} \cap \operatorname{Aff}(\tau)$ has volume 1 . The product $(\operatorname{dim} \tau) ! \operatorname{Vol}(\tau)$ is also called the normalized volume of the face $\tau$. If $\tau$ is a simplicial facet, this normalized 
volume is equal to the multiplicity of the cone spanned by the vertices divided by the 'distance' of the facet to the origin. This distance is the number $N$ in the equation $\sum_{i=1}^{n} a_{i} x_{i}=N$ of $\operatorname{Aff}(\tau)\left(\operatorname{where} \operatorname{gcd}\left(a_{1}, \ldots, a_{n}\right)=1\right)$.

In this last theorem we clearly see that a set of candidate poles (containing all poles) of the local topological zeta function is given by -1 together with the rational numbers $-\nu(a) / N(a)$ for $a$ orthogonal to a facet of the Newton polyhedron at the origin. We say that such a facet contributes the candidate pole.

Remark 1. There is also a graphical way to determine the candidate pole contributed by a facet $\tau$. If $(r, \ldots, r)$ is the intersection point of the diagonal of the first quadrant with the affine hyperplane containing $\tau$, then the candidate pole $-\nu(\tau) / N(\tau)$ is equal to $-1 / r$.

Example 1. Note that $f(x, y)=x^{2} y^{7}+x^{8} y+x^{7} y^{6}+x^{10}+y^{12}$ is nondegenerate with respect to its Newton polyhedron at the origin, so we can use Theorem 10 to compute its local topological zeta function.
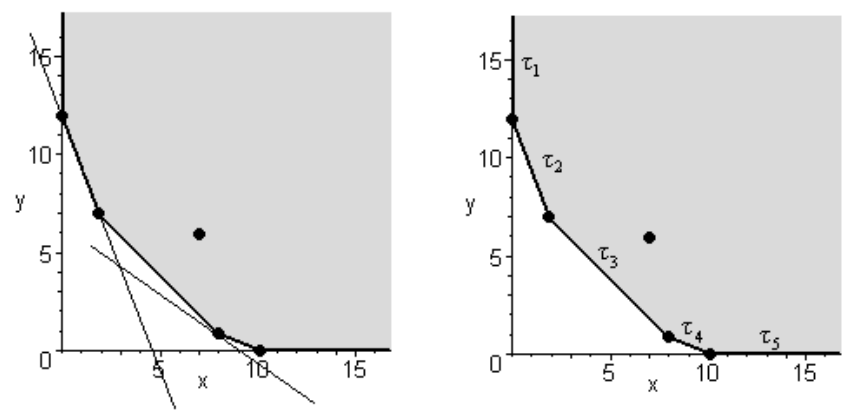

Figure 1

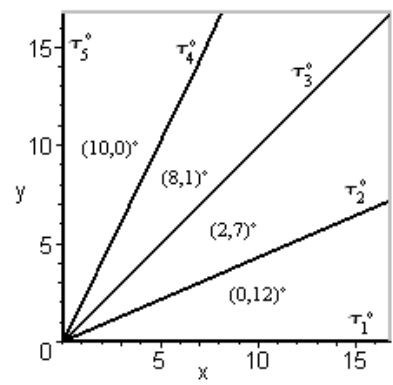

Figure 2

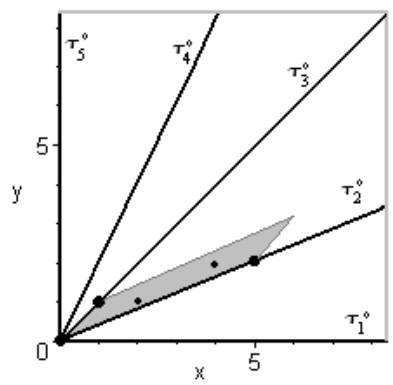

Figure 3

The equations and volumes of the compact facets $\tau_{2}, \tau_{3}$ and $\tau_{4}$ (see Figure 1) are respectively $5 x+2 y=24, \operatorname{Vol}\left(\tau_{2}\right)=1, x+y=9, \operatorname{Vol}\left(\tau_{3}\right)=6$ and $x+2 y=$ $10, \operatorname{Vol}\left(\tau_{4}\right)=1$. The cones dual to the four vertices are all simplicial (see Figure 2 ). We compute that mult $(2,7)^{\circ}=3$ (see Figure 3 ), mult $(0,12)^{\circ}=2$, mult $(8,1)^{\circ}=1$ 
and mult $(10,0)^{\circ}=1$. The local topological zeta function is equal to

$$
\begin{aligned}
Z_{t o p, f}(s)= & \frac{2}{24 s+7}+\frac{3}{(9 s+2)(24 s+7)}+\frac{1}{(9 s+2)(10 s+3)}+\frac{1}{10 s+3} \\
& -\frac{s}{s+1}\left(\frac{1}{24 s+7}+\frac{6}{9 s+2}+\frac{1}{10 s+3}\right) \\
= & -\frac{135 s^{2}-5 s-14}{(s+1)(9 s+2)(24 s+7)} .
\end{aligned}
$$

We now recall the formula for the zeta function of monodromy in the origin in terms of the Newton polyhedron. This was done by Varchenko (see Var76]) and uses the formula of A'Campo (see $\mathrm{A}^{\prime} \mathrm{C} 75$ ). Again, let $f\left(x_{1}, \ldots, x_{n}\right)$ be a polynomial satisfying $f(0)=0$ and suppose $f$ is nondegenerate with respect to its Newton polyhedron $\Gamma_{0}$. Varchenko defines functions $\zeta_{\tau}(t)$ for the compact faces $\tau$ of $\Gamma_{0}$ for which there exists a subset $I \subset\{1, \ldots, n\}$ with $\# I=\operatorname{dim}(\tau)+1$, such that $\tau \subset L_{I}:=\left\{x \in \mathbb{R}^{n} \mid \forall i \notin I: x_{i}=0\right\}$. Let $\sum_{i \in I} a_{i} x_{i}=m$ be the equation of $\operatorname{Aff}(\tau)$ in its corresponding $L_{I}$, where $m$ and all $a_{i}$ (for $i \in I$ ) are positive integers and the greatest common divisor of the $a_{i}, i \in I$, is equal to 1 . We put

$$
\zeta_{\tau}(t):=\left(1-t^{m}\right)^{\operatorname{dim}(\tau) ! \operatorname{Vol}(\tau)} .
$$

Theorem 2 ([Var76]). The zeta function of monodromy of $f$ in the origin is equal to

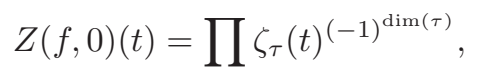

where the product runs over all faces $\tau$ of $\Gamma_{0}$ for which $\zeta_{\tau}$ is defined above.

Example 1 continued. We compute $\zeta_{\tau}(t)$ for $\tau$ equal to the vertices $(10,0)$ and $(0,12)$ and for $\tau$ equal to the compact facets. We get

$$
\zeta_{\tau_{2}}(t)=\left(1-t^{24}\right), \quad \zeta_{\tau_{3}}(t)=\left(1-t^{9}\right)^{6}, \quad \zeta_{\tau_{4}}(t)=\left(1-t^{10}\right) .
$$

For $\tau$ equal to $(10,0)$ or $(0,12)$, the corresponding equations are respectively $x=10$ and $y=12$ and the volumes are both 1 . This results in

$$
\zeta_{(10,0)}(t)=\left(1-t^{10}\right), \quad \zeta_{(0,12)}(t)=\left(1-t^{12}\right) .
$$

The zeta function of monodromy of $f$ in the origin is equal to

$$
Z(f, 0)(t)=\frac{1-t^{12}}{\left(1-t^{24}\right)\left(1-t^{9}\right)^{6}} .
$$

Notice that in the previous example all poles of $Z_{t o p, f}$ induce eigenvalues of monodromy. Indeed, $-3 / 10$ is a false candidate pole. This candidate pole is contributed by what we will call a $B_{1}$-facet. Also, other kinds of facets can yield false candidate poles. The idea of Loeser in L90 was to show that almost all compact facets give rise to an eigenvalue of monodromy. For $\tau$ and $\sigma$ two facets of a Newton polyhedron, he defines $\beta(\tau, \sigma)$ to be the greatest common divisor of the $(2 \times 2)$-minors of the matrix with as rows the relatively prime coefficients of the dual rays $\tau^{\circ}$ and $\sigma^{\circ}$. Then he puts

$$
\lambda(\tau, \sigma)=\nu(\sigma)-\frac{\nu(\tau)}{N(\tau)} N(\sigma) \quad \text { and } \quad \mu(\tau, \sigma)=\frac{\lambda(\tau, \sigma)}{\beta(\tau, \sigma)} .
$$


Using this terminology, his theorem is the following:

Theorem 3 ([L90]). Let $p$ be a prime number and $K$ a finite extension field of $\mathbb{Q}_{p}$. Let $f \in K\left[x_{1}, \ldots, x_{n}\right]$ be a polynomial satisfying $f(0)=0$. Suppose $f$ is nondegenerate with respect to its Newton polyhedron $\Gamma_{0}$ at the origin. Let a compact facet $\tau$ of $\Gamma_{0}$ satisfy the conditions

(1) $\nu(\tau) / N(\tau)$ is no integer;

(2) for every facet $\sigma$ of $\Gamma_{0}$ that is different from $\tau$ and has nonempty intersection with $\tau$, we have that $\mu(\tau, \sigma)$ is no integer.

Then $e^{-2 \pi i \nu(\tau) / N(\tau)}$ is an eigenvalue of monodromy of $f$ at the origin.

Actually, this result is a corollary of the stronger statement he proved about zeroes of the Bernstein-Sato polynomial. Notice that Theorem 3 cannot be applied to facet $\tau_{4}$ of Example 1. In dimension 3 we now want to omit these conditions and also include the poles coming from noncompact facets (such Newton polyhedra typically represent nonisolated singularities).

\section{Determination of a Set of eigenvalues of MONODROMy}

3.1. Strategy. To explain the objective of this section, we introduce the notion of a $B_{1}$-facet. We say that a facet $\tau$ of an $n$-dimensional Newton polyhedron is noncompact for the variable $x_{j}(1 \leq j \leq n)$ if for every point $p \in \tau$ the point $p+(0, \ldots, 0,1,0, \ldots, 0) \in \tau$, where $(0, \ldots, 0,1,0, \ldots, 0)$ is an $n$-tuple with 1 at place $j$ and 0 everywhere else. We define the maps $\pi_{j}: \mathbb{R}^{n} \rightarrow \mathbb{R}^{n-1}:\left(x_{1}, \ldots, x_{n}\right) \mapsto$ $\left(x_{1}, \ldots, \widehat{x_{j}}, \ldots, x_{n}\right)$ for $j=1, \ldots, n$.

Definition 3. A simplicial facet of an $n$-dimensional Newton polyhedron $(n \geq 2)$ is a $B_{1}$-simplex with respect to the variable $x_{i}$ if it is a simplex with $n-1$ vertices in the coordinate hyperplane $x_{i}=0$ and one vertex at distance one from this hyperplane.

A noncompact facet $\tau$ of an $n$-dimensional Newton polyhedron $(n \geq 3)$ is a (noncompact) $B_{1}$-facet with respect to the variable $x_{i}$ if $\tau$ is noncompact for exactly one variable $x_{j}$ and if $\pi_{j}(\tau)$ is a $B_{1}$-simplex in $\mathbb{R}^{n-1}$ with respect to $x_{i}$.

A $B_{1}$-facet is a $B_{1}$-simplex or a noncompact $B_{1}$-facet with respect to some variable.

Throughout this section we are going to prove the following result about facets that induce eigenvalues of monodromy.

Theorem 4. Let $f$ be a complex polynomial in two or three variables. Suppose that $f$ is nondegenerate with respect to its Newton polyhedron $\Gamma_{0}$ at the origin. Then for every facet $\tau$ of $\Gamma_{0}$ that is not a $B_{1}$-facet, the complex number $e^{-2 \pi i \nu(\tau) / N(\tau)}$ is an eigenvalue of monodromy of $f$ at a point in the neighbourhood of the origin.

The idea of the proof is the following. Remember that the zeta function of monodromy associated to an isolated singularity in odd dimensions is a polynomial; in even dimensions it is $(1-t)$ divided by a polynomial. Indeed, for isolated singularities, Milnor proved that the cohomology groups $H^{i}(F, \mathbb{C})$ are $\{0\}$ for $i \neq 0$ and $i \neq n-1$, where $n$ denotes the dimension (see Mi68).

We will group the factors $\zeta_{\tau}$ as defined in Section 2. For a compact facet $\tau$ of a two-dimensional Newton polyhedron, we define $F_{\tau}$ as

$$
F_{\tau}:=\left(\zeta_{\tau}\right)^{-1} \prod \zeta_{p}
$$


where the product runs over the vertices $p \in \tau$ that lie on coordinate axes. Then we see that in dimension 2 , if the Newton polyhedron contains a compact facet, the zeta function of monodromy at the origin is equal to

$$
Z(f, 0)(t)=\prod_{\tau \text { a compact facet }} F_{\tau} .
$$

In Subsection 3.2, we will show that such a factor $F_{\tau}$ is the inverse of a polynomial (except when $\tau$ contains two vertices on the coordinate axes). This implies that a pole of such an $F_{\tau}$ is also a pole of the monodromy zeta function at the origin.

If we work with a three-dimensional Newton polyhedron, we first partition every compact facet into simplices. For such a simplex $\tau$ we define the factor $F_{\tau}$ as follows:

$$
F_{\tau}:=\zeta_{\tau} \prod_{\sigma}\left(\zeta_{\sigma}\right)^{-1} \prod_{p} \zeta_{p}
$$

where the first product runs over the line segments $\sigma$ in $\tau$ that lie in coordinate planes and the second product runs over vertices $p$ of $\tau$ that are intersection points of two such line segments (and hence lie on a coordinate axis). Note that for a partition of a compact facet $\tau$ into simplices $\tau_{1}, \ldots, \tau_{r}$ we have that $\zeta_{\tau}=\prod_{i=1}^{r} \zeta_{\tau_{i}}$. Following the formula of Varchenko, the zeta function of monodromy at the origin is

$$
Z(f, 0)(t)=\prod_{\tau} F_{\tau} \prod_{\sigma}\left(\zeta_{\sigma}\right)^{-1} \prod_{p} \zeta_{p},
$$

where the first product runs over the compact simplices obtained after subdividing the compact facets $\tau$ and the other products run over line segments $\sigma$ in coordinate hyperplanes and vertices $p$ on coordinate axes for which $\zeta_{\sigma}$, respectively $\zeta_{p}$, was not used in any $F_{\tau}$. We will show that every $F_{\tau}$ is a polynomial and that for every compact (simplicial) facet $\tau$ that is not a $B_{1}$-simplex, the complex number $e^{-2 \pi i \nu(\tau) / N(\tau)}$ is a zero of $F_{\tau}$. When the Newton polyhedron does not contain any line segment in a coordinate plane that is not included in a compact facet, then every compact facet that is not a $B_{1}$-simplex induces an eigenvalue of monodromy at the origin. When the Newton polyhedron does not satisfy this condition, we might need to consider the zeta function of monodromy at a point in the neighbourhood of the origin. Also for a noncompact facet that is not a $B_{1}$-facet we consider the zeta function of monodromy in a point different from the origin to conclude that it induces an eigenvalue of monodromy. Lemma 9 is the key lemma to handle these situations.

3.2. Monodromy eigenvalues in dimension 2. In this subsection we explore the situation in dimension 2 . Note that then the monodromy conjecture has already been proven by Loeser (see L88]). However, the result about facets that induces eigenvalues of monodromy in dimension 2 will be needed later on when we work in dimension 3 .

Proposition 5. Let $\tau$ be a compact simplex of some two-dimensional Newton polyhedron. If $\tau$ does not contain two vertices on coordinate axes, then $F_{\tau}$ is the inverse of a polynomial. If moreover $\tau$ is not a $B_{1}$-simplex, then $e^{-2 \pi i \nu(\tau) / N(\tau)}$ is a pole of $F_{\tau}$. If $\tau$ contains two vertices on coordinate axes, if $\tau$ is not a $B_{1}$-simplex and $-\nu(\tau) / N(\tau) \neq-1$, then $e^{-2 \pi i \nu(\tau) / N(\tau)}$ is a pole of $F_{\tau}$. 
Proof. First suppose that $\tau$ does not contain any vertex on a coordinate axis. Then $F_{\tau}=\left(\zeta_{\tau}\right)^{-1}$ and the number $e^{-2 \pi i \nu(\tau) / N(\tau)}$ is obviously a pole.

Then suppose that $\tau$ contains exactly one vertex on a coordinate axis. We introduce the integers $k, l, m$ as in Figure 4 and put $g:=\operatorname{gcd}(k-m, l)$. Then $g$ is

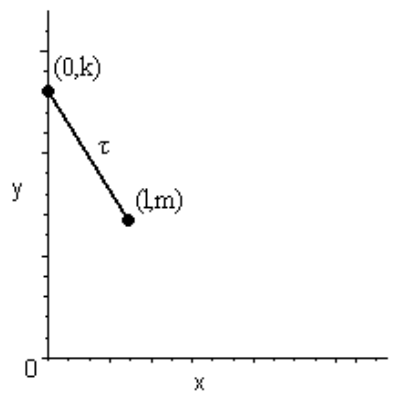

Figure 4

the (normalized) volume of the facet $\tau$,

$$
\operatorname{Aff}(\tau) \leftrightarrow \frac{k-m}{g} x+\frac{l}{g} y=\frac{l k}{g} \quad \text { and } \quad F_{\tau}=\frac{\left(1-t^{k}\right)}{\left(1-t^{l k / g}\right)^{g}} .
$$

Since $g$ divides $l, F_{\tau}$ is obviously the inverse of a polynomial. We need to verify if under the condition $l>1$, the rational number $s_{0}=-\frac{(l+k-m) / g}{l k / g}$ induces a pole $e^{2 \pi i s_{0}}$ of $F_{\tau}$. If $g>1$, this is obvious. If $g=1$, it is only false if $e^{2 \pi i s_{0}}$ is a $k$ th root of unity. This is equivalent to $l$ being a divisor of $k-m$, which can only be true if $l=1$.

Now suppose $\tau$ contains two vertices on coordinate axes. Note that then the zeta function of monodromy is equal to $F_{\tau}$. Suppose the vertices are $(l, 0)$ and $(0, k)$ and suppose $g=\operatorname{gcd}(l, k)$. Then the supporting hyperplane of $\tau$ has equation $(k / g) x+(l / g) y=l k / g$ and

$$
F_{\tau}=\frac{\left(1-t^{l}\right)\left(1-t^{k}\right)}{\left(1-t^{l k / g}\right)^{g}}
$$

If $g>2$, it is obvious that $s_{0}=-\frac{(l+k) / g}{l k / g}$ induces a pole of $F_{\tau}$. If $g=2$, this would only be false if $e^{2 \pi i s_{0}}$ is an $l$ th and a $k$ th root of unity. This means respectively that $k / 2$ and $l / 2$ are divisors of $(l+k) / 2$, so $l=k=2$ and $s_{0}=-1$. This case was excluded. If $g=1$, the complex number $e^{-2 \pi i(l+k) / l k}$ is a pole unless $l$ or $k$ divides $l+k$, but since $g=1$, this implies that $l$ or $k$ is 1 and $\tau$ is a $B_{1}$-simplex.

The following theorem is Theorem 4 for a polynomial in two variables.

Theorem 6. Let $f(x, y)$ be a complex polynomial that is nondegenerate with respect to its Newton polyhedron $\Gamma_{0}$. If $s_{0}=-\nu(\tau) / N(\tau)$ for a facet $\tau$ that is not a $B_{1^{-}}$ simplex and not contained in a coordinate axis, then $e^{2 \pi i s_{0}}$ is an eigenvalue of monodromy of $f$ at a point in the neighbourhood of the origin.

Proof. If the facet $\tau$ is compact, this now follows immediately from Proposition 5 (taking into account that 1 is always an eigenvalue of monodromy). 
Suppose $\tau$ is not compact. Then $\operatorname{Aff}(\tau) \leftrightarrow x=a$ for $a \in \mathbb{Z}_{>0}$ and $s_{0}=-1 / a$. We choose $k \in \mathbb{C} \backslash\{0\}$ and show that $e^{-2 \pi i / a}$ is an eigenvalue of monodromy at the point $(0, k)$. Therefore we need the Newton polyhedron of $f(x, y)$ at $(0, k)$, which is equal to the Newton polyhedron of the polynomial $f(x, y-k)$ at the origin. For almost all $k$ this polyhedron only has one vertex, the point $(a, 0)$, and thus the polynomial $f(x, y-k)$ is then nondegenerate with respect to its Newton polyhedron in the origin. So we can use the formula of Varchenko to compute the zeta function of monodromy of $f$ at $(0, k)$. This zeta function is equal to $\left(1-t^{a}\right)$. We conclude that $e^{-2 \pi i / a}$ is an eigenvalue of monodromy of $f$ at the point $(0, k)$.

3.3. Monodromy eigenvalues in dimension 3. To prepare the proof of Theorem 4 in dimension 3, we first show Proposition 8 and Lemma 9. Lemma 9 is our key tool to handle nonisolated singularities. We will use the following property on greatest common divisors.

Lemma 7. Let $a, b, c$ and $d$ be positive integer numbers. Then

$$
\operatorname{gcd}(a, b) \operatorname{gcd}(c, d)=\operatorname{gcd}(a c, a d, b c, b d) .
$$

Proposition 8. Let $\tau$ be a compact simplex of some three-dimensional Newton polyhedron. Then $F_{\tau}$ is a polynomial. Moreover, if $\tau$ is not a $B_{1}$-simplex, then $e^{-2 \pi i \nu(\tau) / N(\tau)}$ is a zero of $F_{\tau}$.

Proof.

Case 1. $\tau$ does not contain a segment in a coordinate plane.

If the 2-simplex $\tau$ in $\Gamma_{0}$ does not contain a segment that is lying in a coordinate plane, then $F_{\tau}=\zeta_{\tau}$. For such a facet $\tau$ we clearly have that $e^{-2 \pi i \nu(\tau) / N(\tau)}$ is a zero of $F_{\tau}$.

Case 2. $\tau$ contains exactly one segment in a coordinate plane.

Part A. As illustrated in Figure 5, let $\tau$ be the facet with vertices with coordinates $(a, b, c),(0, k, l)$ and $(0, m, n)$ and let $\sigma$ be the segment with vertices $(0, k, l)$ and $(0, m, n)$. The equation of $\operatorname{Aff}(\tau)$ is given by

$$
x(k(c-n)+b(n-l)+m(l-c))+y(a(l-n))+z(a(m-k))=a(m l-k n) .
$$

(The integers $k, n, b$ and $c$ might be zero, but not in a combination that introduces a new line segment in a coordinate plane.) Let $g_{\tau}$ be the greatest common divisor of the coefficients:

$$
\begin{aligned}
g_{\tau} & :=\operatorname{gcd}(k(c-n)+b(n-l)+m(l-c), a(l-n), a(m-k)) \\
& =\operatorname{gcd}((l-n)(m-b)+(m-k)(n-c), a(l-n), a(m-k)) .
\end{aligned}
$$

The normalized volume of a facet can be computed by putting its vertices in a matrix and dividing the determinant of this matrix by the distance $N(\tau)$. Hence we find $(\operatorname{dim} \tau) ! \operatorname{Vol}(\tau)=g_{\tau}$ and $\zeta_{\tau}(t)=\left(1-t^{\frac{a(m l-k n)}{g_{\tau}}}\right)^{g_{\tau}}$.

The segment $\sigma$ has as equation, in the coordinate plane $x=0$,

$$
y(a(l-n))+z(a(m-k))=a(m l-k n) .
$$




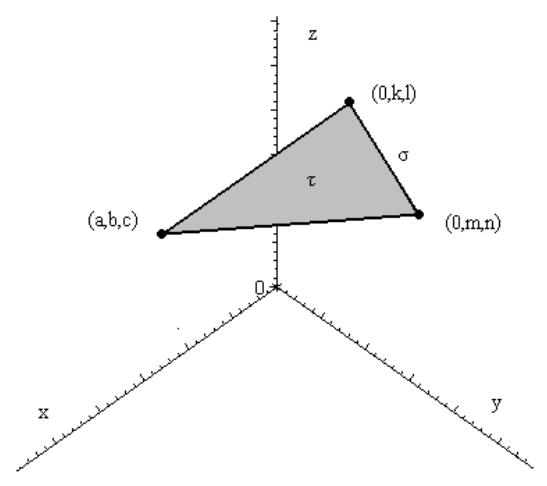

Figure 5

Let $g_{\sigma}:=\operatorname{gcd}\left(\frac{a(l-n)}{g_{\tau}}, \frac{a(m-k)}{g_{\tau}}\right)$. The normalized volume $(\operatorname{dim} \sigma) ! \operatorname{Vol}(\sigma)$ of $\sigma$ is equal to $\frac{g_{\tau} g_{\sigma}}{a}=\operatorname{gcd}(l-n, m-k)$ and thus $\zeta_{\sigma}(t)=\left(1-t^{\frac{a(m l-k n)}{g_{\tau} g_{\sigma}}}\right)^{\frac{g_{\tau} g_{\sigma}}{a}}$. We get

$$
F_{\tau}=\frac{\left(1-t^{\frac{a(m l-k n)}{g_{\tau}}}\right)^{g_{\tau}}}{\left(1-t^{\frac{a(m l-k n)}{g_{\tau} g_{\sigma}}}\right)^{\frac{g_{\tau} g_{\sigma}}{a}} .}
$$

As $\frac{g_{\tau} g_{\sigma}}{a}$ divides $l-n$ and $m-k$, it also divides $g_{\tau}$. This shows that $F_{\tau}$ is a polynomial.

Part B. We will now show that $e^{-2 \pi i \nu(\tau) / N(\tau)}$ is a zero of $F_{\tau}$. The candidate pole $-\nu(\tau) / N(\tau)$ is equal to

$$
-\frac{(k(c-n)+b(n-l)+m(l-c)+a(l-n)+a(m-k)) / g_{\tau}}{a(m l-k n) / g_{\tau}} .
$$

This rational number would not induce a zero of $F_{\tau}$ if

$$
g_{\sigma}=a \text { and } g_{\tau} g_{\sigma} \mid k(c-n)+b(n-l)+m(l-c)+a(l-n)+a(m-k) .
$$

Since $g_{\tau} g_{\sigma}$ is a divisor of the last two terms, it follows that $g_{\sigma}$ must be equal to 1 to avoid a contradiction with the definition of $g_{\tau}$. So also $a=1$ and $\tau$ is a $B_{1}$-simplex.

We conclude that when $\tau$ is not a $B_{1}$-simplex, then $e^{-2 \pi i \nu(\tau) / N(\tau)}$ is a zero of $F_{\tau}$. When $\tau$ is a $B_{1}$-simplex (so $\left.a=1\right)$, it can easily be seen that $g_{\tau}=\operatorname{gcd}(l-n, m-k$ ). This implies that $g_{\sigma}=1$ and $F_{\tau}=1$.

Case 3. $\tau$ contains exactly two segments in coordinate planes.

Part A. Let $\tau$ be the facet with vertices $(a, 0, c),(0,0, l)$ and $(0, m, n)$. Let $\sigma_{1}$ be the segment with vertices $(0,0, l)$ and $(0, m, n)$ and let $\sigma_{2}$ be the segment with vertices $(0,0, l)$ and $(a, 0, c)$, as illustrated in Figure 6 . We first show that $F_{\tau}$ is a polynomial. The equation of $\operatorname{Aff}(\tau)$ is given by

$$
x(m(l-c))+y(a(l-n))+z a m=a m l .
$$

(One of the integers $n$ or $c$ might be zero, but not both.) Let $g_{\tau}$ be the greatest common divisor of the coefficients: $g_{\tau}:=\operatorname{gcd}(m(l-c), a(l-n), a m)$. We find $(\operatorname{dim} \tau) ! \operatorname{Vol}(\tau)=g_{\tau}$ and hence $\zeta_{\tau}(t)=\left(1-t^{\frac{a m l}{g_{\tau}}}\right)^{g_{\tau}}$. 


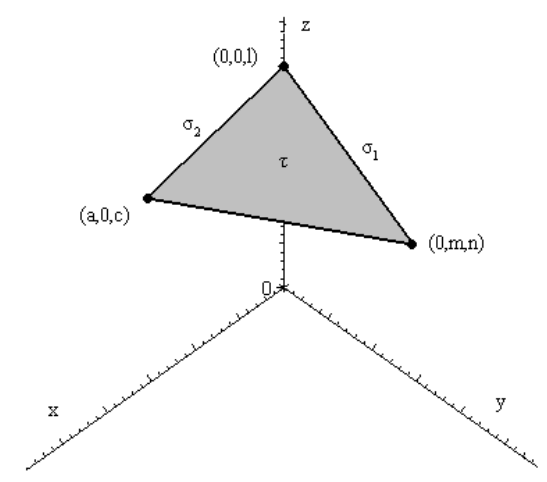

Figure 6

The segment $\sigma_{1}$ has as equation, in the coordinate plane $x=0$,

$$
y(a(l-n))+z a m=a m l .
$$

Let $g_{\sigma_{1}}:=\operatorname{gcd}\left(\frac{a(l-n)}{g_{\tau}}, \frac{a m}{g_{\tau}}\right)$. The normalized volume of $\sigma$ is equal to $\frac{g_{\tau} g_{\sigma_{1}}}{a}=$ $\operatorname{gcd}(l-n, m)$ and thus $\zeta_{\sigma_{1}}(t)=\left(1-t^{\frac{a m l}{g_{\tau} g_{\sigma_{1}}}}\right)^{\frac{g_{\tau} g_{\sigma_{1}}}{a}}$. Analogously for the segment $\sigma_{2}$ we find that $\zeta_{\sigma_{2}}(t)=\left(1-t^{\frac{a m l}{g_{\tau} g_{2}}}\right)^{\frac{g_{\tau} g_{\sigma_{2}}}{m}}$, with $\frac{g_{\tau} g_{\sigma_{2}}}{m}=\operatorname{gcd}(l-c, a)$.

Let $p$ be the intersection point of the segments $\sigma_{1}$ and $\sigma_{2}$. Then $\zeta_{p}(t)=\left(1-t^{l}\right)$. We then get that

$$
F_{\tau}=\frac{\left(1-t^{l}\right)\left(1-t^{\frac{a m l}{g_{\tau}}}\right)^{g_{\tau}}}{\left(1-t^{\frac{a m l}{g_{\tau} g_{\sigma_{1}}}}\right)^{\frac{g_{\tau} g_{\sigma_{1}}}{a}}\left(1-t^{\frac{a m l}{g_{\tau} g_{2}}}\right)^{\frac{g_{\tau} g_{\sigma_{2}}}{m}}} .
$$

By using Lemma 7 we can conclude that always

$$
\begin{aligned}
\frac{g_{\tau} g_{\sigma_{1}}}{a}+\frac{g_{\tau} g_{\sigma_{2}}}{m} & \leq \operatorname{gcd}(l-n, m) \cdot \operatorname{gcd}(l-c, a)+1 \\
& \leq g_{\tau}+1
\end{aligned}
$$

and that

$$
\frac{g_{\tau} g_{\sigma_{1}}}{a}+\frac{g_{\tau} g_{\sigma_{2}}}{m} \leq g_{\tau}
$$

whenever $\frac{g_{\tau} g_{\sigma_{1}}}{a} \neq 1$ and $\frac{g_{\tau} g_{\sigma_{2}}}{m} \neq 1$. If $g_{\tau} \geq \frac{g_{\tau} g_{\sigma_{1}}}{a}+\frac{g_{\tau} g_{\sigma_{2}}}{m}$, then clearly $F_{\tau}$ is a polynomial. Now suppose that $\frac{g_{\tau} g_{\sigma_{1}}}{a}+\frac{g_{\tau} g_{\sigma_{2}}}{m}=g_{\tau}+1$ and that $\frac{g_{\tau} g_{\sigma_{1}}}{a}=$ $\operatorname{gcd}(l-n, m)=1$ (which also implies that $g_{\sigma_{2}}=m$ ). To show that $F_{\tau}$ is a polynomial, we prove that a root of both factors in the denominator is also a root of $\left(1-t^{l}\right)$. We show that $\operatorname{gcd}\left(\frac{a m l}{g_{\tau} g_{\sigma_{1}}}, \frac{a m l}{g_{\tau} g_{\sigma_{2}}}\right)=l$. Now $\operatorname{gcd}\left(\frac{a m}{g_{\tau} g_{\sigma_{1}}}, \frac{a m}{g_{\tau} g_{\sigma_{2}}}\right)=\operatorname{gcd}\left(m, a / g_{\tau}\right)=$ $\operatorname{gcd}\left(g_{\sigma_{2}}, g_{\sigma_{1}}\right)$ and this last greatest common divisor is equal to 1 by definition of $g_{\tau}$.

Part B. Now we investigate when $e^{-2 \pi i \nu(\tau) / N(\tau)}$ is a zero of $F_{\tau}$. When $\tau$ is a $B_{1^{-}}$ simplex, we may suppose $a=1$. This implies $g_{\tau}=\operatorname{gcd}(l-n, m), g_{\sigma_{1}}=1$ and $\frac{g_{\tau} g_{\sigma_{2}}}{m}=1$, which leads to $F_{\tau}=1$. So from now on, we suppose $a, m>1$. The candidate pole $s_{0}=-\nu(\tau) / N(\tau)$ is equal to

$$
-\frac{(m(l-c)+a(l-n)+a m) / g_{\tau}}{a m l / g_{\tau}} .
$$


The number $e^{-2 \pi i \nu(\tau) / N(\tau)}$ is certainly a zero of $F_{\tau}$ if $g_{\tau}>\frac{g_{\tau} g_{\sigma_{1}}}{a}+\frac{g_{\tau} g_{\sigma_{2}}}{m}$. Let us see what happens when $g_{\tau} \leq \frac{g_{\tau} g_{\sigma_{1}}}{a}+\frac{g_{\tau} g_{\sigma_{2}}}{m}$. One can check from the definitions of $g_{\tau}$ and $g_{\sigma_{1}}$ that $e^{2 \pi i s_{0}}$ is a zero of the first factor of the denominator if and only if $g_{\sigma_{1}}=1$. We also have the analogous statement for the second factor. Since $a, m>1$ the number $e^{2 \pi i s_{0}}$ is a zero of $F_{\tau}$ if this number is a zero of at most one of the two factors of the denominator. If $g_{\sigma_{1}}=g_{\sigma_{2}}=1$ we are in the situation $g_{\tau} \leq \frac{g_{\tau}}{a}+\frac{g_{\tau}}{m}$. So $a=m=2$. First note that then $g_{\tau} / 2=\operatorname{gcd}(l-n, 2)$ and $g_{\tau} / 2=\operatorname{gcd}(l-c, 2)$. We conclude that $\operatorname{gcd}(l-n, 2)=\operatorname{gcd}(l-c, 2)$ and thus $(l-n)+(l-c)$ is even. The candidate pole $s_{0}$ is equal to

$$
-\frac{2(l-c)+2(l-n)+4}{4 l} .
$$

Since the numerator is divisible by 4 , the number $e^{2 \pi i s_{0}}$ is a zero of $\left(1-t^{l}\right)$, which proves our result.

We conclude that when $\tau$ is not a $B_{1}$-simplex, then $e^{-2 \pi i \nu(\tau) / N(\tau)}$ is a zero of $F_{\tau}$. When $\tau$ is a $B_{1}$-simplex, then $F_{\tau}=1$.

Case 4. $\tau$ contains three segments in coordinate planes.

Part A. Let $\tau$ be the facet with vertices $(a, 0,0),(0,0, l)$ and $(0, m, 0)$. Let $\sigma_{1}$ be the segment with vertices $(0,0, l)$ and $(0, m, 0)$, let $\sigma_{2}$ be the segment with vertices $(0,0, l)$ and $(a, 0,0)$ and let $\sigma_{3}$ be the segment with vertices $(0, m, 0)$ and $(a, 0,0)$. This notation is illustrated in Figure 7.

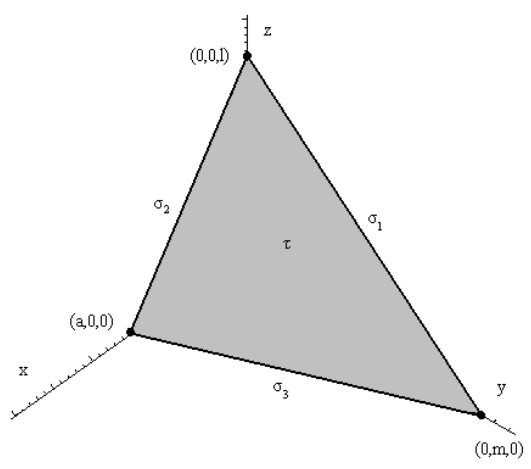

FiguRE 7

The equation of $\tau$ is given by

$$
x(m l)+y(a l)+z(a m)=a m l .
$$

We define $g_{\tau}, g_{\sigma_{1}}, g_{\sigma_{2}}$ and $g_{\sigma_{3}}$ such that

(3)

$$
g_{\tau}=\operatorname{gcd}(m l, a l, a m), \quad \frac{g_{\tau} g_{\sigma_{1}}}{a}=\operatorname{gcd}(l, m), \quad \frac{g_{\tau} g_{\sigma_{2}}}{l}=\operatorname{gcd}(a, m), \quad \frac{g_{\tau} g_{\sigma_{3}}}{m}=\operatorname{gcd}(a, l) .
$$

We then get that

$$
F_{\tau}=\frac{\left(1-t^{l}\right)\left(1-t^{m}\right)\left(1-t^{a}\right)\left(1-t^{\frac{a m l}{g_{\tau}}}\right)^{g_{\tau}}}{\left(1-t^{\frac{a m l}{g_{\tau} g_{\sigma_{1}}}}\right)^{\frac{g_{\tau} g_{\sigma_{1}}}{a}}\left(1-t^{\frac{a m l}{g_{\tau} g_{\sigma_{2}}}}\right)^{\frac{g_{\tau} g_{\sigma_{2}}}{l}}\left(1-t^{\frac{a m l}{g_{\tau} g_{\sigma_{3}}}}\right)^{\frac{g_{\tau} g_{\sigma_{3}}}{m}}} .
$$


This is certainly a polynomial, since it is equal to the zeta function of monodromy of an isolated singularity.

Part B. If $a, m$ or $l$ equals 1 , the facet is a $B_{1}$-simplex. Suppose $a=1$. Then $g_{\tau}=\operatorname{gcd}(l, m)$, which implies $g_{\sigma_{1}}=1$. Moreover we know $\frac{g_{\tau} g_{\sigma_{2}}}{l}=\frac{g_{\tau} g_{\sigma_{3}}}{m}=1$. All this leads to $F_{\tau}=1-t$. Note that this could be known in advance, since in this case $\Gamma_{0}$ is the Newton polyhedron of a polynomial with a regular point at the origin. From now on, we suppose $a, l, m>1$. We investigate whether $e^{-2 \pi i \nu(\tau) / N(\tau)}$ is always a zero of $F_{\tau}$. The candidate pole $s_{0}=-\nu(\tau) / N(\tau)$ is equal to

$$
-\frac{(l m+a l+a m) / g_{\tau}}{a m l / g_{\tau}}=-\left(\frac{1}{a}+\frac{1}{m}+\frac{1}{l}\right) .
$$

When $g_{\tau}>\frac{g_{\tau} g_{\sigma_{1}}}{a}+\frac{g_{\tau} g_{\sigma_{2}}}{l}+\frac{g_{\tau} g_{\sigma_{3}}}{m}$, then $e^{2 \pi i s_{0}}$ is certainly a zero of $F_{\tau}$. First we claim for $j=1,2,3$ : the complex number $e^{2 \pi i s_{0}}$ is a zero of the $j$ th factor of the denominator if and only if $g_{\sigma_{j}}=1$. This follows from the definitions of the greatest common divisors. Now suppose that $g_{\tau}=\frac{g_{\tau} g_{\sigma_{1}}}{a}+\frac{g_{\tau} g_{\sigma_{2}}}{l}+\frac{g_{\tau} g_{\sigma_{3}}}{m}$. It can only happen that $e^{2 \pi i s_{0}}$ is not a zero of $F_{\tau}$ if $e^{2 \pi i s_{0}}$ is a zero of the three factors in the denominator. This implies that $g_{\sigma_{1}}=g_{\sigma_{2}}=g_{\sigma_{3}}=1$. On the other hand, we then have $\frac{1}{a}+\frac{1}{l}+\frac{1}{m}=1$ and this means that $e^{2 \pi i s_{0}}=1$ is a zero of every factor in the numerator. In this way we find that $e^{2 \pi i s_{0}}$ is a (multiple) zero of $F_{\tau}$. So we are left with the case that $g_{\tau}<\frac{g_{\tau} g_{\sigma_{1}}}{a}+\frac{g_{\tau} g_{\sigma_{2}}}{l}+\frac{g_{\tau} g_{\sigma_{3}}}{m}$. If $e^{2 \pi i s_{0}}$ is not a zero of any factor of the denominator, then we are done. If only $g_{\sigma_{1}}=1$, then we use $a>1$ to conclude that $e^{2 \pi i s_{0}}$ is a zero of $F_{\tau}$. So we also suppose $g_{\sigma_{2}}=1$.

- If $g_{\sigma_{3}} \neq 1$, the only situation where $e^{2 \pi i s_{0}}$ might be canceled as a zero of $F_{\tau}$ occurs when $g_{\tau} \leq g_{\tau} / a+g_{\tau} / l$. This implies $a=l=2$ and $g_{\tau}=g_{\tau} / a+g_{\tau} / l$. In this case we find $s_{0}=-\frac{m+1}{m}$ and moreover we see that $e^{2 \pi i s_{0}}$ is a zero of the factor $\left(1-t^{m}\right)$ of the numerator.

- If also $g_{\sigma_{3}}=1$, we have $1<\frac{1}{a}+\frac{1}{l}+\frac{1}{m}$. We may suppose $a=2$. The equations (3) are now

$$
g_{\tau}=2 \operatorname{gcd}(l, m), \quad g_{\tau}=l \operatorname{gcd}(2, m), \quad g_{\tau}=m \operatorname{gcd}(2, l) .
$$

We can easily see that $l$ and $m$ cannot both be odd. So suppose $l$ is even. If also $m$ is even, then $g_{\tau}=2 m=2 l$. We know that then the condition $1<\frac{1}{2}+\frac{2}{m}$ should be satisfied. Since $m$ is even, we are in the situation $a=m=l=2$. One calculates that then

$$
F_{\tau}=\frac{\left(1-t^{2}\right)^{3}\left(1-t^{2}\right)^{4}}{\left(1-t^{2}\right)^{2}\left(1-t^{2}\right)^{2}\left(1-t^{2}\right)^{2}}=1-t^{2}
$$

which leads to the fact that $e^{2 \pi i s_{0}}=e^{-3 \pi i}=-1$ is a zero of $F_{\tau}$.

If $m$ is odd, we have $g_{\tau}=2 m=l$. In this case we can calculate

$$
\begin{aligned}
F_{\tau} & =\frac{\left(1-t^{2 m}\right)\left(1-t^{m}\right)\left(1-t^{2}\right)\left(1-t^{2 m}\right)^{2 m}}{\left(1-t^{2 m}\right)^{m}\left(1-t^{2 m}\right)\left(1-t^{2 m}\right)^{2}} \\
& =\left(1-t^{m}\right)\left(1-t^{2}\right)\left(1-t^{2 m}\right)^{m-2} .
\end{aligned}
$$

Since $m \geq 3$, the candidate pole $s_{0}=-\frac{m+3}{2 m}$ induces a zero $e^{2 \pi i s_{0}}$ of $F_{\tau}$.

We conclude that $e^{-2 \pi i \nu(\tau) / N(\tau)}$ is a zero of $F_{\tau}$, except when $\tau$ is a $B_{1}$-simplex. In that case, $F_{\tau}=(1-t)$. 
To prove Theorem 4 for $f$ a polynomial in three variables, we need the following lemma about nondegeneracy.

Lemma 9. If a complex polynomial $f(x, y, z)$ is nondegenerate with respect to its Newton polyhedron at the origin, then for almost all $k \in \mathbb{C}$ the polynomial $f(x, y, z-k)$ is nondegenerate with respect to its Newton polyhedron at the origin. (An analogous result holds for the variables $x$ and $y$.)

Proof. We will denote the Newton polyhedron of $f(x, y, z-k)$, for $k \in \mathbb{C}$, by $\Gamma_{k}$. Notice that for almost all $k$, the polyhedron $\Gamma_{k}$ is the projection of $\Gamma_{0}$ on the plane $z=0$ times $\mathbb{R}_{\geq 0}$ and thus is the same for these $k$.

Suppose we have an infinite number of values of $k$ for which the polynomial $f(x, y, z)$ is degenerate with respect to its Newton polyhedron at the point $(0,0, k)$. As the Newton polyhedron only has a finite number of faces, there exists a compact line segment $\sigma$ of $\Gamma_{k}$ such that there are infinitely many $k \in \mathbb{C}$ for which $g_{\sigma}$ has a singular point outside the coordinate axes. We now also consider $k$ as a variable, so we look at $g_{\sigma} \in \mathbb{C}[x, y, k]$. This equation is quasi-homogeneous in $x$ and $y$. If $\operatorname{Aff}(\sigma) \leftrightarrow b x+a y=c$, then we substitute $x$ by $u^{a}$ and $y$ by $v^{b}$. Then $g_{\sigma}(u, v, k)$ is homogeneous in $u$ and $v$. We dehomogenize by setting $v=1$ and we decompose $g_{\sigma}(u, 1, k)$ into its irreducible components: $g_{\sigma}(u, 1, k)=\prod_{i=1}^{p} g_{i}^{m_{i}}(u, 1, k)$. As there are infinitely many values for $k$ for which $g_{\sigma}(u, 1, k)$ has a singular point outside $u=0$, it follows that there exists at least one component $g_{i} \in\left\{g_{1}, \ldots, g_{p}\right\}$ that is not a monomial and for which the exponent $m_{i}$ is greater than or equal to 2 . Say that $g_{1}$ satisfies these conditions. We now homogenize again and we substitute $u^{a}$ by $x$ and $v^{b}$ by $y$. If we substitute $k$ in $g_{\sigma}(x, y, k)$ by the variable $z$, then for the noncompact facet $\tau^{\prime}$ of $\Gamma_{0}$ that projects onto $\sigma$ we have that $f_{\tau^{\prime}}(x, y, z)=$ $g_{\sigma}(x, y, z)=\prod_{i=1}^{p} g_{i}^{m_{i}}(x, y, z)$. This notation is illustrated in Figure 8.

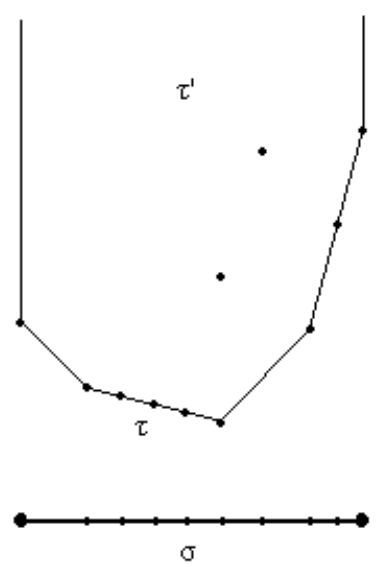

Figure 8

Let us now look at the factor $g_{1}^{m_{1}}(x, y, z)$ that is quasi-homogeneous in $x$ and $y$. We write $g_{1}=M_{1}+\cdots+M_{d}$ with the $M_{i}, 1 \leq i \leq d$, monomials in $\mathbb{C}[x, y, z]$. Let $N_{1}, \ldots, N_{e}$ be the monomials in $\mathbb{C}[x, y, z]$ such that $f_{\tau^{\prime}}=g_{1}^{m_{1}}\left(N_{1}+\cdots+N_{e}\right)$. We can choose $T \subset\{1, \ldots, d\}$, \#T $\geq 2$, such that for every $i \in\{1, \ldots, e\}$, the 
support of $\left\{N_{i} M_{t}^{m_{1}}\right\}_{t \in T}$ is contained in a line $\ell_{i}$ and such that all support points of $\left\{N_{i} M_{1}^{m_{1}}, \ldots, N_{i} M_{d}^{m_{1}}\right\} \backslash\left\{N_{i} M_{t}^{m_{1}}\right\}_{t \in T}$ lie above $\ell_{i}$. Note that $\ell_{1}, \ldots, \ell_{e}$ are parallel. Let $\ell$ be the lowest of the lines $\ell_{1}, \ldots, \ell_{e}$ and suppose $\ell_{i_{1}}, \ldots, \ell_{i_{j}} \subset\left\{\ell_{1}, \ldots, \ell_{e}\right\}$ coincide with $\ell$. The support points of $f(x, y, z)$ on $\ell$ are exactly the support points of the polynomial $\left(N_{i_{1}}+\cdots+N_{i_{j}}\right)\left(\sum_{t \in T} M_{t}\right)^{m_{1}}$. The line segment containing these support points is a compact face $\tau$ of $\Gamma_{0}$. The polynomial $f_{\tau}=\left(N_{i_{1}}+\cdots+\right.$ $\left.N_{i_{j}}\right)\left(\sum_{t \in T} M_{t}\right)^{m_{1}}$ has a singular point outside the coordinate planes. This implies that $f$ is degenerate with respect to $\Gamma_{0}$ and leads to a contradiction.

We can now prove Theorem 4 for a polynomial in three variables.

Theorem 10. Let $f$ be a complex polynomial in three variables. Suppose that $f$ is nondegenerate with respect to its Newton polyhedron $\Gamma_{0}$ at the origin. Then for every facet $\tau$ of $\Gamma_{0}$ that is not a $B_{1}$-facet, the complex number $e^{-2 \pi i \nu(\tau) / N(\tau)}$ is an eigenvalue of monodromy of $f$ at a point in the neighbourhood of the origin.

Proof. First suppose $\tau$ is compact. If every line segment of the Newton polyhedron that is contained in a coordinate plane is also contained in a compact facet, then we know from equation (2) and Proposition 8 that the zeta function of monodromy at the origin is a product of polynomials.

If $\tau$ is a compact facet that is not a union of $B_{1}$-simplices, then Proposition 8 says that $e^{-2 \pi i \nu(\tau) / N(\tau)}$ is a root of it. If $\tau$ is a compact facet which is a union of different $B_{1}$-simplices, then, up to permutation of the coordinates, the facet $\tau$ should have vertices $(a, 0,0),(c, d, 0),(b, 1,1)$ and $(e, 0, f)$ for $a, \ldots, f \in \mathbb{Z}_{\geq 0}$. One can verify that the candidate pole contributed by $\tau$ is equal to $-(1-b+a) / a$. The factor $\zeta_{(a, 0,0)}=\left(1-t^{a}\right)$ was not used in any $F_{\tau}$. From equality (2) we deduce that $e^{-2 \pi i(1-b+a) / a}$ is an eigenvalue of monodromy in the origin.

Let $\sigma$ be a line segment in a coordinate plane, say in $z=0$, but which is not contained in any compact facet. We suppose moreover that $e^{-2 \pi i \nu(\tau) / N(\tau)}$ is a pole of $F_{\sigma}$, where we use the notation $F_{\sigma}$ as if $\sigma$ was a facet of a two-dimensional Newton polyhedron in the plane $z=0$. (Thus $F_{\sigma}=\left(\zeta_{\sigma}\right)^{-1}$ may be multiplied with $\zeta_{p}$ if $\sigma$ contains a point $p$ on a coordinate axis.) If $\sigma$ would be a $B_{1}$-simplex in the coordinate plane $z=0$, then $F_{\sigma}=1$, so this is also excluded. Now we choose a $k \in \mathbb{C}$ close to zero such that $f(x, y, z+k)$ is nondegenerate with respect to its Newton polyhedron in the origin (see Lemma 9). The support points of this new polynomial are the projections on the plane $z=0$ of the old support points. So the monodromy zeta function of $f(x, y, z)$ at the point $(0,0,-k)$ is equal to the monodromy zeta function of a polynomial in two variables with this projected polyhedron as its Newton polyhedron. From Proposition 5 we deduce that a pole of $F_{\sigma}$ is also a pole of this monodromy zeta function.

Now suppose that $\tau$ is not compact, say for the variable $z$, and that it is not a $B_{1}$-facet. So it has an equation of the form $a x+b y=N$ for $a, b, N \in \mathbb{Z}$. Then we choose again a value $k \in \mathbb{C}$ for which $f(x, y, z+k)$ is nondegenerate with respect to its Newton polyhedron at the origin. Analogously as in the previous paragraph, we use the zeta function of monodromy of a polynomial in two variables. We know that there is a facet of the two-dimensional Newton polyhedron that has equation $a x+b y=N$. Since this facet is not a $B_{1}$-simplex, we conclude from Theorem 6 that $e^{-2 \pi i \nu(\tau) / N(\tau)}$ is an eigenvalue of monodromy of $f$ at a point in the neighbourhood of $(0,0,-k)$. 


\section{The MONODRomy CONJECTURE FOR NONDEGENERATE SINGULARITIES IN DIMENSIONS 2 AND 3}

4.1. On false poles contributed by facets and the monodromy conjecture in dimension 2. The poles of the local topological zeta function can be determined out of the resolution graph (see Ve95, Theorem 4.3]). In [LVa09] we showed that, for nondegenerate plane curves, these poles are not so special in the sense that they are not selected. Indeed, all facets that are not $B_{1}$-simplices induce a pole of the local topological zeta function. Maybe this also offers some explanation why relations with for instance jumping numbers are not so surprising in dimension 2 .

Theorem 11 ([LVa09, Theorem 3]). Let $f$ be a complex polynomial in two variables. Suppose that $f$ is nondegenerate with respect to its Newton polyhedron $\Gamma_{0}$. Then for a candidate pole $s_{0} \neq-1$ contributed by some facet, the following holds:

$$
s_{0} \text { is a pole of } Z_{\text {top }, f}
$$

品

$s_{0}$ is contributed by a facet that is not a $B_{1}$-simplex.

Theorem [6 and Theorem 11 easily lead to a proof of the monodromy conjecture for nondegenerate polynomials in two variables.

Theorem 12. Let $f(x, y)$ be a complex polynomial that is nondegenerate with respect to its Newton polyhedron at the origin. If $s_{0}$ is a pole of the local topological zeta function of $f$, then $e^{2 \pi i s_{0}}$ is an eigenvalue of monodromy of $f$ at some point of $\{f=0\}$ close to 0 .

4.2. On false poles contributed by facets in dimension 3. In Subsection 3.3 we proved that all facets, except the $B_{1}$-facets, induce eigenvalues of monodromy at a point in the neighbourhood of the origin. With the monodromy conjecture in mind, we will now investigate candidate poles contributed by $B_{1}$-facets. We will show that in almost all cases candidate poles of the local topological zeta function that are only contributed by $B_{1}$-simplices are not actual poles. Indeed, it will seem that there is only one exception in dimension 3 , namely a candidate pole contributed by various $B_{1}$-facets only with respect to different variables having a line segment in common. We will come back to this later. Note that we will not mind skipping the candidate pole -1 , since we know that $e^{-2 \pi i}=1$ is always an eigenvalue of monodromy.

Denef proved the following theorem (with unpublished proof) about these $B_{1}$ simplices:

Theorem 13. Let $f$ be a polynomial over $\mathbb{Z}_{p}$ in $n$ variables. Suppose that $f$ is nondegenerate over $\mathbb{Q}_{p}$ with respect to the compact faces of its Newton polyhedron. Suppose that $s_{0} \neq-1$ is the real part of a candidate pole of Igusa's local zeta function such that $s_{0}$ is only contributed by one $B_{1}$-simplex. Then there is no pole of the p-adic Igusa zeta function with real part equal to $s_{0}$.

We prove the following proposition about candidate poles contributed by $B_{1}$-facets of a three-dimensional Newton polyhedron.

Proposition 14. Let $f$ be a complex polynomial in three variables that is nondegenerate with respect to its Newton polyhedron. Suppose $s_{0} \neq-1$ is a candidate pole of the local topological zeta function only contributed by facets that are $B_{1}$-facets 
with respect to the same variable or by $B_{1}$-facets with respect to possibly different variables having an intersection of dimension at most zero. Then $s_{0}$ is not a pole.

Proof. We will consider seven separate cases. We investigate all possible configurations of $B_{1}$-facets (compact and noncompact) contributing the same candidate pole $s_{0} \neq-1$.

Case $1 . s_{0}$ is contributed by exactly one $B_{1}$-simplex.

Suppose $s_{0}$ is only contributed by the $B_{1}$-simplex $\tau$. We fix notation as in Figure 9. The vertices $A$ and $B$ of $\tau$ are contained in the coordinate plane $z=0$. The

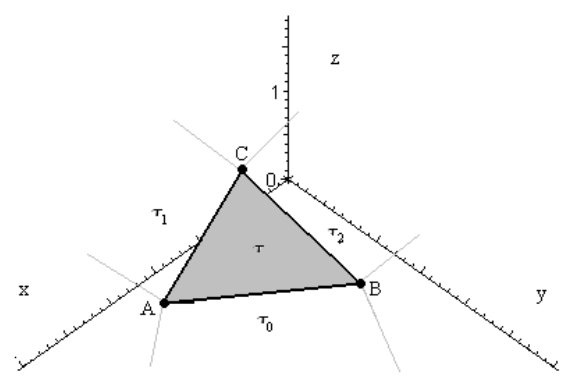

Figure 9

vertex $C$ lies at distance one. The other facets that contain the line segments $A B, A C$ and $B C$ are respectively $\tau_{0}, \tau_{1}$ and $\tau_{2}$. The facet $\tau_{0}$ is not compact and is contained in the hyperplane $z=0$. There might be other facets containing the vertices $A, B$ or $C$. If for instance $A$ is contained in more than three facets, the dual cone $A^{\circ}$ is not simplicial. However, we can take a subdivision of $A^{\circ}$ containing the cone $\Delta_{A}$ which we define as the cone spanned by the rays $\tau_{0}^{\circ}, \tau^{\circ}$ and $\tau_{1}^{\circ}$. This is now the only simplicial cone in the subdivision of $A^{\circ}$ that contains $\tau^{\circ}$. The simplicial cones $\Delta_{B}$ and $\Delta_{C}$ are defined analogously. We introduce the following notation:

$$
\begin{aligned}
\operatorname{Aff}(\tau) & \leftrightarrow a_{\tau}^{x} x+a_{\tau}^{y} y+a_{\tau}^{z} z=N(\tau), \\
\operatorname{Aff}\left(\tau_{1}\right) & \leftrightarrow a_{\tau_{1}}^{x} x+a_{\tau_{1}}^{y} y+a_{\tau_{1}}^{z} z=N\left(\tau_{1}\right), \\
\operatorname{Aff}\left(\tau_{2}\right) & \leftrightarrow a_{\tau_{2}}^{x} x+a_{\tau_{2}}^{y} y+a_{\tau_{2}}^{z} z=N\left(\tau_{2}\right),
\end{aligned}
$$

where we suppose that the coefficients of the equations of the facets are relatively prime. Note that $\nu(\tau)=a_{\tau}^{x}+a_{\tau}^{y}+a_{\tau}^{z}, \nu\left(\tau_{1}\right)=a_{\tau_{1}}^{x}+a_{\tau_{1}}^{y}+a_{\tau_{1}}^{z}$ and $\nu\left(\tau_{2}\right)=a_{\tau_{2}}^{x}+a_{\tau_{2}}^{y}+$ $a_{\tau_{2}}^{z}$. Following Theorem 1 we need to consider the following terms of the topological zeta function and prove that $-\nu(\tau) / N(\tau)$ is not a pole of this sum:

$$
\begin{aligned}
& \frac{\operatorname{mult}\left(\Delta_{A}\right)}{(N(\tau) s+\nu(\tau))\left(N\left(\tau_{1}\right) s+\nu\left(\tau_{1}\right)\right)}+\frac{\operatorname{mult}\left(\Delta_{B}\right)}{(N(\tau) s+\nu(\tau))\left(N\left(\tau_{2}\right) s+\nu\left(\tau_{2}\right)\right)} \\
& +\frac{\operatorname{mult}\left(\Delta_{C}\right)}{(N(\tau) s+\nu(\tau))\left(N\left(\tau_{2}\right) s+\nu\left(\tau_{2}\right)\right)\left(N\left(\tau_{1}\right) s+\nu\left(\tau_{1}\right)\right)} \\
& -\frac{s}{(s+1)} \frac{\operatorname{mult}\left(A C^{\circ}\right) \operatorname{Vol}(A C)}{(N(\tau) s+\nu(\tau))\left(N\left(\tau_{1}\right) s+\nu\left(\tau_{1}\right)\right)}-\frac{s}{(s+1)} \frac{\operatorname{mult}\left(B C^{\circ}\right) \operatorname{Vol}(B C)}{(N(\tau) s+\nu(\tau))\left(N\left(\tau_{2}\right) s+\nu\left(\tau_{2}\right)\right)} \\
& -\frac{s}{(s+1)} \frac{\operatorname{mult}\left(A B^{\circ}\right) \operatorname{Vol}(A B)}{(N(\tau) s+\nu(\tau))}+\frac{s}{(s+1)} \frac{\operatorname{mult}\left(\tau^{\circ}\right) 2 ! \operatorname{Vol}(\tau)}{(N(\tau) s+\nu(\tau))} .
\end{aligned}
$$


To simplify this expression, we need a few easy results.

i) $\operatorname{gcd}\left(a_{\tau}^{x}, a_{\tau}^{y}\right)=1, \operatorname{gcd}\left(a_{\tau_{1}}^{x}, a_{\tau_{1}}^{y}\right)=1, \operatorname{gcd}\left(a_{\tau_{2}}^{x}, a_{\tau_{2}}^{y}\right)=1$. The three facets $\tau, \tau_{1}$, $\tau_{2}$ all contain two points of the form $(\cdot, \cdot, 0)$ and $(\cdot, \cdot, 1)$. Putting these in the equations shows that there exist integers $k, l, m, n$ such that

$$
a_{\tau}^{z}=k a_{\tau}^{x}+l a_{\tau}^{y}, \quad a_{\tau_{1}}^{z}=k a_{\tau_{1}}^{x}+l a_{\tau_{1}}^{y}, \quad a_{\tau}^{z}=m a_{\tau}^{x}+n a_{\tau}^{y}, \quad a_{\tau_{2}}^{z}=m a_{\tau_{2}}^{x}+n a_{\tau_{2}}^{y} .
$$

So a common divisor of the first two coefficients is also a divisor of the third one. Since the three coefficients are relatively prime, this leads to this first result.

ii) $\operatorname{mult}\left(\tau^{\circ}\right)=\operatorname{mult}\left(A B^{\circ}\right)=1$. For the first multiplicity it is obvious that the multiplicity is equal to one. For the second, one can use the previous item.

iii) $2 ! \operatorname{Vol}(\tau)=\operatorname{Vol}(A B)$. The normalized volume of $\tau$ is equal to the quotient of the determinant of the matrix with the vertices and $N(\tau)$. The normalized volume of $A B$ can be computed in the hyperplane $z=0$. So we need the determinant of the matrix with the first two entries of $A$ and $B$ and we divide this by the constant term of the equation of $A B$ in $z=0$. We use the first result again to see that this constant term is equal to $N(\tau)$. The two mentioned determinants are obviously equal.

iv) $\operatorname{Vol}(A C)=\operatorname{Vol}(B C)=1$. This is immediate from the definition of volume.

v) $\operatorname{mult}\left(\Delta_{A}\right)=\operatorname{mult}\left(A C^{\circ}\right), \operatorname{mult}\left(\Delta_{B}\right)=\operatorname{mult}\left(B C^{\circ}\right)$. We can compute the multiplicity of $\Delta_{A}$ as

$$
\operatorname{mult}\left(\Delta_{A}\right)=\left|\begin{array}{ccc}
0 & 0 & 1 \\
a_{\tau_{1}}^{x} & a_{\tau_{1}}^{y} & a_{\tau_{1}}^{z} \\
a_{\tau}^{x} & a_{\tau}^{y} & a_{\tau}^{z}
\end{array}\right|
$$

Using the equalities stated in $\mathbf{i}$ ), this is equal to

$$
\operatorname{mult}\left(A C^{\circ}\right)=\operatorname{gcd}\left(\left|\begin{array}{cc}
a_{\tau_{1}}^{x} & a_{\tau_{1}}^{y} \\
a_{\tau}^{x} & a_{\tau}^{y}
\end{array}\right|,\left|\begin{array}{cc}
a_{\tau_{1}}^{x} & a_{\tau_{1}}^{z} \\
a_{\tau}^{x} & a_{\tau}^{z}
\end{array}\right|,\left|\begin{array}{cc}
a_{\tau_{1}}^{y} & a_{\tau_{1}}^{z} \\
a_{\tau}^{y} & a_{\tau}^{z}
\end{array}\right|\right) .
$$

The second equality is analogous.

We use all this to simplify the part of the local topological zeta function written above and find:

$$
\begin{aligned}
& \frac{\operatorname{mult}\left(\Delta_{A}\right)}{(s+1)(N(\tau) s+\nu(\tau))\left(N\left(\tau_{1}\right) s+\nu\left(\tau_{1}\right)\right)}+\frac{\operatorname{mult}\left(\Delta_{B}\right)}{(s+1)(N(\tau) s+\nu(\tau))\left(N\left(\tau_{2}\right) s+\nu\left(\tau_{2}\right)\right)} \\
& +\frac{\operatorname{mult}\left(\Delta_{C}\right)}{(N(\tau) s+\nu(\tau))\left(N\left(\tau_{2}\right) s+\nu\left(\tau_{2}\right)\right)\left(N\left(\tau_{1}\right) s+\nu\left(\tau_{1}\right)\right)} .
\end{aligned}
$$

To end the proof of this first case, one has to show that $N(\tau) s+\nu(\tau)$ is a divisor of

$$
\operatorname{mult}\left(\Delta_{A}\right)\left(N\left(\tau_{2}\right) s+\nu\left(\tau_{2}\right)\right)+\operatorname{mult}\left(\Delta_{B}\right)\left(N\left(\tau_{1}\right) s+\nu\left(\tau_{1}\right)\right)+\operatorname{mult}\left(\Delta_{C}\right)(s+1) .
$$

This can be done by explicit computations using a computer algebra package.

Case 2. $s_{0}$ is contributed by exactly one $B_{1}$-facet and this $B_{1}$-facet is not compact.

Suppose $s_{0}$ is only contributed by the noncompact $B_{1}$-facet $\tau$. Say that $\tau$ is noncompact for the variable $z$ and that it is a $B_{1}$-facet with respect to the variable $y$. We fix notation as in Figure 10.

The vertex $A$ is contained in the coordinate plane $y=0$ and the vertex $B$ is lying at a distance one from the hyperplane $y=0$. We define the facet $\tau_{1}$ as the facet that has the segment $A B$ in common with $\tau$. The noncompact facet that contains the vertex $B$ and that has a half-line in common with $\tau$ will be called $\tau_{2}$. 


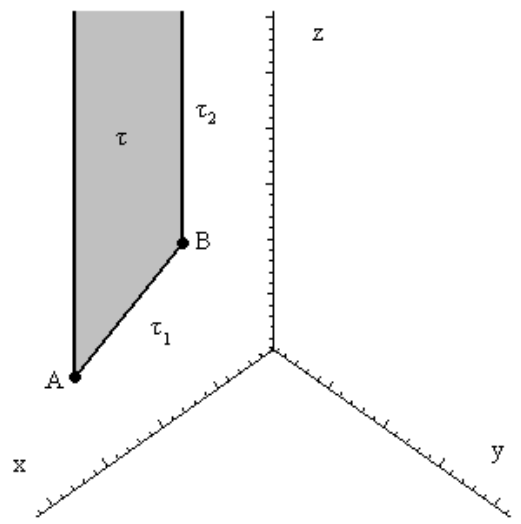

FiguRE 10

There might be other facets that contain the vertices $A$ or $B$. However, we can investigate whether $-\nu(\tau) / N(\tau)$ is a pole of $Z_{\text {top }, f}$ by taking only the terms of $Z_{\text {top }, f}$ into account which are provided by the cone $A B^{\circ}$, by the cone $\Delta_{A}$ spanned by the rays $\tau^{\circ},(0,1,0), \tau_{1}^{\circ}$ and by the cone $\Delta_{B}$ spanned by the rays $\tau^{\circ}, \tau_{1}^{\circ}$ and $\tau_{2}^{\circ}$ :

$$
\begin{aligned}
& -\frac{s}{(s+1)} \frac{\operatorname{mult}\left(A B^{\circ}\right) \operatorname{Vol}(A B)}{(N(\tau) s+\nu(\tau))\left(N\left(\tau_{1}\right) s+\nu\left(\tau_{1}\right)\right)}+\frac{\operatorname{mult}\left(\Delta_{A}\right)}{(N(\tau) s+\nu(\tau))\left(N\left(\tau_{1}\right) s+\nu\left(\tau_{1}\right)\right)} \\
& +\frac{\operatorname{mult}\left(\Delta_{B}\right)}{(N(\tau) s+\nu(\tau))\left(N\left(\tau_{2}\right) s+\nu\left(\tau_{2}\right)\right)\left(N\left(\tau_{1}\right) s+\nu\left(\tau_{1}\right)\right)} .
\end{aligned}
$$

As in Case 1 one finds that $\operatorname{mult}\left(\Delta_{A}\right)=\operatorname{mult}\left(A B^{\circ}\right)$ and $\operatorname{Vol}(A B)=1$. If we set $A(k, 0, l)$ and $B(m, 1, n)$ and if we write

$$
\begin{aligned}
\operatorname{Aff}(\tau) & \leftrightarrow x+(k-m) y=k \\
\operatorname{Aff}\left(\tau_{1}\right) & \leftrightarrow a_{\tau_{1}}^{x} x+a_{\tau_{1}}^{y} y+a_{\tau_{1}}^{z} z=a_{\tau_{1}}^{x} k+a_{\tau_{1}}^{z} l=a_{\tau_{1}}^{x} m+a_{\tau_{1}}^{y}+a_{\tau_{1}}^{z} n, \\
\operatorname{Aff}\left(\tau_{2}\right) & \leftrightarrow a x+b y=a m+b
\end{aligned}
$$

with $\operatorname{gcd}\left(a_{\tau_{1}}^{x}, a_{\tau_{1}}^{y}, a_{\tau_{1}}^{z}\right)=1=\operatorname{gcd}(a, b)$, then one also easily computes that $\operatorname{mult}\left(\Delta_{A}\right)$ $=a_{\tau_{1}}^{z}$ and mult $\left(\Delta_{B}\right)=a_{\tau_{1}}^{z}(a(k-m)-b)$. The contribution to the topological zeta function written above then becomes

$$
\frac{a_{\tau_{1}}^{z}\left(N\left(\tau_{2}\right) s+\nu\left(\tau_{2}\right)\right)+a_{\tau_{1}}^{z}(a(k-m)-b)(s+1)}{(s+1)(N(\tau) s+\nu(\tau))\left(N\left(\tau_{1}\right) s+\nu\left(\tau_{1}\right)\right)\left(N\left(\tau_{2}\right) s+\nu\left(\tau_{2}\right)\right)} .
$$

This can be simplified to

$$
\frac{a a_{\tau_{1}}^{z}}{(s+1)\left(N\left(\tau_{1}\right) s+\nu\left(\tau_{1}\right)\right)\left(N\left(\tau_{2}\right) s+\nu\left(\tau_{2}\right)\right)},
$$

which implies that $-\nu(\tau) / N(\tau)$ is not a pole.

Case $3 . s_{0}$ is contributed by exactly two $B_{1}$-simplices with respect to the same variable, having a line segment in common.

Suppose $s_{0}$ is only contributed by the $B_{1}$-simplices $\tau$ and $\sigma$. We fix notation as in Figure 11. The vertices $A, B$ and $C$ are contained in the coordinate plane $z=0$. The vertex $D$ lies at distance one. The other facets that contain the line 


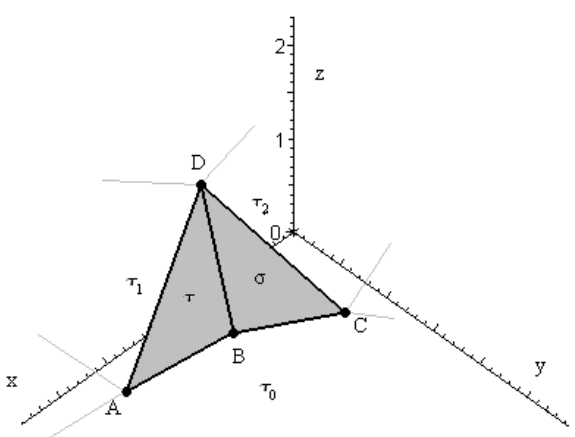

FIGURE 11

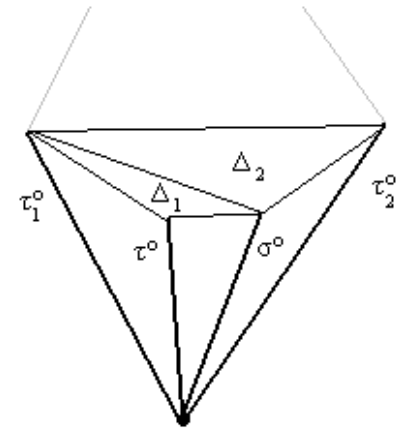

FigURE 12

segments $A D$ and $C D$ are respectively $\tau_{1}$ and $\tau_{2}$. The facet $\tau_{0}$ is not compact and is contained in the hyperplane $z=0$. For $A^{\circ}$ and $C^{\circ}$ we use an analogous subdivision as in Case 1. So we define $\Delta_{A}$ as the cone spanned by the rays $\tau_{1}^{\circ}, \tau^{\circ}$ and $\tau_{0}^{\circ}$ and $\Delta_{C}$ as the cone spanned by the rays $\tau_{2}^{\circ}, \sigma^{\circ}$ and $\tau_{0}^{\circ}$. The cone $B^{\circ}$ is simplicial, so $\Delta_{B}:=B^{\circ}$. The vertex $D$ is contained in at least four facets. We define $\Delta_{1}$ as the cone spanned by $\tau_{1}^{\circ}, \tau^{\circ}$ and $\sigma^{\circ}$ and $\Delta_{2}$ as the cone spanned by $\sigma^{\circ}, \tau_{1}^{\circ}$ and $\tau_{2}^{\circ}$. This can be seen in Figure 12. Now the proof goes further in the same way as in Case 1. The terms in the local topological zeta function originating from $A B$ and $\tau$ cancel. So do the terms coming from $B C$ and $\sigma$. We again find that $\operatorname{mult}\left(\Delta_{A}\right)=\operatorname{mult}\left(A D^{\circ}\right), \operatorname{mult}\left(\Delta_{B}\right)=\operatorname{mult}\left(B D^{\circ}\right)$ and $\operatorname{mult}\left(\Delta_{C}\right)=\operatorname{mult}\left(C D^{\circ}\right)$. The relevant contribution to the topological zeta function is thus given by

$$
\begin{aligned}
& \frac{\operatorname{mult}\left(\Delta_{A}\right)}{(s+1)(N(\tau) s+\nu(\tau))\left(N\left(\tau_{1}\right) s+\nu\left(\tau_{1}\right)\right)}+\frac{\operatorname{mult}\left(\Delta_{B}\right)}{(s+1)(N(\tau) s+\nu(\tau))(N(\sigma) s+\nu(\sigma))} \\
& +\frac{\operatorname{mult}\left(\Delta_{C}\right)}{(s+1)(N(\sigma) s+\nu(\sigma))\left(N\left(\tau_{2}\right) s+\nu\left(\tau_{2}\right)\right)} \\
& +\frac{\operatorname{mult}\left(\Delta_{1}\right)}{(N(\tau) s+\nu(\tau))\left(N\left(\tau_{1}\right) s+\nu\left(\tau_{1}\right)\right)(N(\sigma) s+\nu(\sigma))} \\
& +\frac{\operatorname{mult}\left(\Delta_{2}\right)}{\left(N\left(\tau_{2}\right) s+\nu\left(\tau_{2}\right)\right)\left(N\left(\tau_{1}\right) s+\nu\left(\tau_{1}\right)\right)(N(\sigma) s+\nu(\sigma))} .
\end{aligned}
$$

To conclude, one can calculate (using a computer algebra package) that the factors $(N(\tau) s+\nu(\tau))(N(\sigma) s+\nu(\sigma))$ disappear when putting everything over the same denominator.

Case $4 . s_{0}$ is contributed by exactly two noncompact $B_{1}$-facets with respect to the same variable, having a line segment in common.

Suppose $s_{0}$ is only contributed by the $B_{1}$-facets $\tau$ and $\sigma$. Since $\sigma$ and $\tau$ are $B_{1}$-facets with respect to the same variable, it is impossible that they have a noncompact half-line in common. We suppose $\tau$ to be noncompact for $z$ and to be a $B_{1}$-facet for $y$. Let the segment $A B$ be the intersection of $\tau$ and $\sigma$.

- If the $B_{1}$-facet $\sigma$ is noncompact for $y$, then to be in the situation of Case 4 we have to ask that $\tau$ and $\sigma$ both are $B_{1}$-facets for the variable $x$. The situation is pictured in Figure 13. Then $A(1,0, k)$ and $B(0,1, l)$ for $k, l \in \mathbb{Z}_{\geq 0}$. The equations of the planes through $\tau$ and $\sigma$ are

$$
\operatorname{Aff}(\tau) \leftrightarrow x+y=1, \quad \operatorname{Aff}(\sigma) \leftrightarrow(l-k) x+z=l .
$$




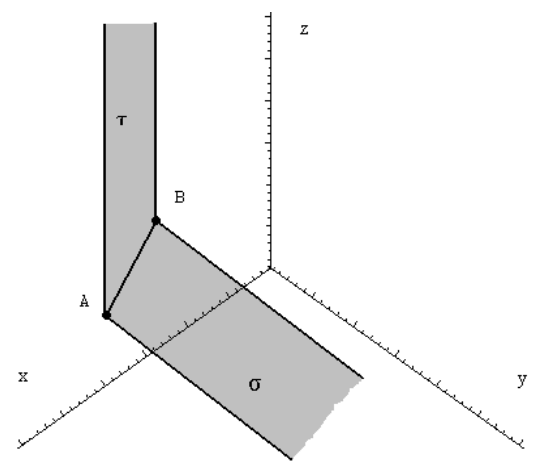

FiguRE 13

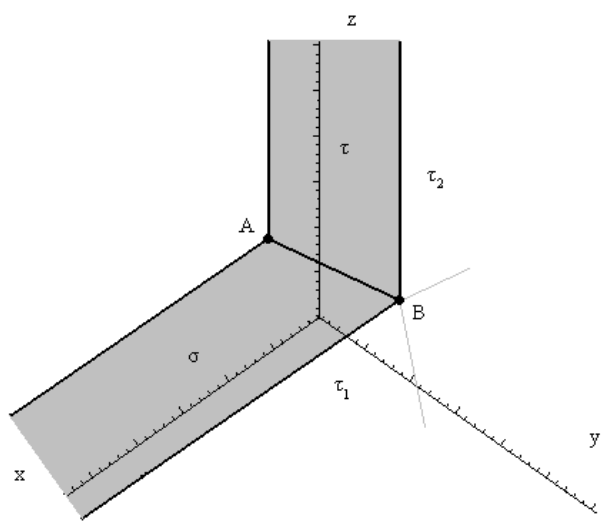

FiguRE 14

Moreover, as $\tau$ and $\sigma$ contribute the same candidate pole, one has that $l=1$ and $k=0$. This implies that the origin is not a singular point of $\{f=0\}$ and thus $-\nu(\tau) / N(\tau)=-2$ is not a pole of $Z_{t o p, f}$.

- If the $B_{1}$-facet $\sigma$ is noncompact for $x$, then the situation is as in Figure 14 . We introduce $k, l, m, n \in \mathbb{Z}_{\geq 0}$ such that $A(k, 0, l)$ and $B(m, 1, n)$. Let $\tau_{1}$ be the noncompact facet containing the vertex $B$ and sharing a half-line with $\sigma$ and let $\tau_{2}$ be the noncompact facet containing the vertex $B$ and sharing a half-line with $\tau$. Let $\Delta_{A}$ be the cone generated by the rays $(0,1,0), \tau^{\circ}, \sigma^{\circ}$, let $\Delta_{B 1}$ be the cone spanned by the rays $\tau^{\circ}, \sigma^{\circ}, \tau_{2}^{\circ}$, and let $\Delta_{B 2}$ be the cone spanned by the rays $\tau_{1}^{\circ}, \sigma^{\circ}, \tau_{2}^{\circ}$. The relevant contribution to the topological zeta function is given by

$$
\begin{gathered}
\frac{-s \text { mult }\left(A B^{\circ}\right) \operatorname{Vol}(A B)}{(s+1)(N(\tau) s+\nu(\tau))(N(\sigma) s+\nu(\sigma))}+\frac{\operatorname{mult}\left(\Delta_{A}\right)}{(N(\tau) s+\nu(\tau))(N(\sigma) s+\nu(\sigma))} \\
+\frac{\operatorname{mult}\left(\Delta_{B 1}\right)}{(N(\tau) s+\nu(\tau))(N(\sigma) s+\nu(\sigma))\left(N\left(\tau_{2}\right) s+\nu\left(\tau_{2}\right)\right)} \\
+\frac{\operatorname{mult}\left(\Delta_{B 2}\right)}{\left(N\left(\tau_{2}\right) s+\nu\left(\tau_{2}\right)\right)\left(N\left(\tau_{1}\right) s+\nu\left(\tau_{1}\right)\right)(N(\sigma) s+\nu(\sigma))} .
\end{gathered}
$$


As in Case 1 we deduce that $\operatorname{mult}\left(A B^{\circ}\right)=\operatorname{mult}\left(\Delta_{A}\right)=1$ and $\operatorname{Vol}(A B)=1$. Moreover, $\tau$ and $\sigma$ give rise to the same candidate pole; hence $k(n-1)=$ $l(m-1)$. If $m=1$, then also $n=1$ (since we have $k \geq m$ ) and then $\nu(\tau) / N(\tau)=1$. So now suppose that $m \neq 1$. Replacing $l$ by $k(n-1) /(m-$ $1)$, we find that in the above expression $(N(\tau) s+\nu(\tau))(N(\sigma) s+\nu(\sigma))$ disappears when putting all terms over the same denominator.

Case 5. $s_{0}$ is contributed by exactly two $B_{1}$-facets (one of them is compact and the other one is not compact) with respect to the same variable, having a line segment in common.

Suppose that the facet $\tau$ is not compact for $z$ and is a $B_{1}$-facet with respect to the variable $y$. Suppose that $\sigma$ is a $B_{1}$-simplex that intersects $\tau$ in the segment $A B$ and that induces the same candidate pole as $\tau$. The facet $\sigma$ should be a $B_{1}$-simplex with respect to $x$ or to $y$ in order to be in Case 5 .

- Suppose that $\sigma$ is a $B_{1}$-simplex for $x$. Then the situation is as in Figure 15 ,

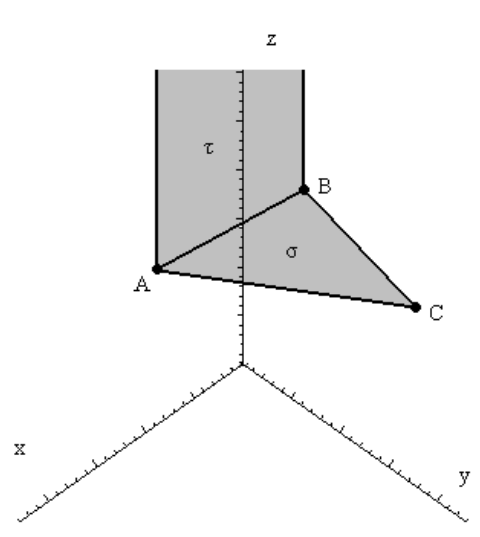

FiguRe 15

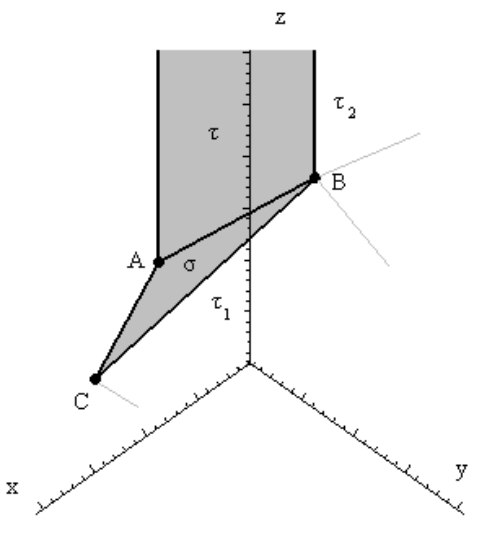

FiguRE 16

with $A(1,0, k), B(0,1, l)$ and $C(0, r, t)$. As $\tau$ and $\sigma$ induce the same candidate pole, we find that $k+l=1$ and thus the origin is not a singular point of $\{f=0\}$ and the only pole of the local topological zeta function is -1 .

- Suppose that $\sigma$ is a $B_{1}$-simplex for $y$. This situation is represented in Figure 16.

We take $k, l, m, n, r, t \in \mathbb{Z}_{\geq 0}$ such that $A(k, 0, l), B(m, 1, n)$ and $C(r, 0, t)$. Let $\tau_{2}$ be the facet containing the vertex $B$ and having a half-line in common with $\tau$. Let $\tau_{1}$ be a facet that intersects $\sigma$ in the line segment $B C$. We 
write

$$
\begin{aligned}
\operatorname{Aff}(\tau) \leftrightarrow & x+(k-m) y=k, \\
\operatorname{Aff}(\sigma) \leftrightarrow & a_{\sigma}^{x} x+a_{\sigma}^{y} y+a_{\sigma}^{z} z=a_{\sigma}^{x} k+a_{\sigma}^{z} l \\
& =a_{\sigma}^{x} m+a_{\sigma}^{y}+a_{\sigma}^{z} n \\
& =a_{\sigma}^{x} r+a_{\sigma}^{z} t \\
\operatorname{Aff}\left(\tau_{1}\right) \leftrightarrow & c x+d y+e z=c m+d+e n=c r+e t, \\
\operatorname{Aff}\left(\tau_{2}\right) \leftrightarrow & a x+b y=a m+b
\end{aligned}
$$

with $\operatorname{gcd}\left(a_{\sigma}^{x}, a_{\sigma}^{y}, a_{\sigma}^{z}\right)=\operatorname{gcd}(c, d, e)=\operatorname{gcd}(a, b)=1$.

Let $\Delta_{A}$ be the cone generated by the rays $(0,1,0), \tau^{\circ}, \sigma^{\circ}$, let $\Delta_{B 1}$ be the cone spanned by the rays $\tau^{\circ}, \sigma^{\circ}, \tau_{2}^{\circ}$, let $\Delta_{B 2}$ be the cone spanned by the rays $\tau_{1}^{\circ}, \sigma^{\circ}, \tau_{2}^{\circ}$ and let $\Delta_{C}$ be the cone spanned by $\tau_{1}^{\circ}, \sigma^{\circ},(0,1,0)$.

The relevant contribution to the topological zeta function is

$$
\begin{aligned}
& \frac{2 \operatorname{Vol}(\sigma) s}{(s+1)(N(\sigma) s+\nu(\sigma))}-\frac{s \operatorname{mult}\left(A B^{\circ}\right) \operatorname{Vol}(A B)}{(s+1)(N(\tau) s+\nu(\tau))(N(\sigma) s+\nu(\sigma))} \\
- & \frac{s \operatorname{mult}\left(B C^{\circ}\right) \operatorname{Vol}(B C)}{(s+1)\left(N\left(\tau_{1}\right) s+\nu\left(\tau_{1}\right)\right)(N(\sigma) s+\nu(\sigma))}-\frac{s \operatorname{mult}\left(A C^{\circ}\right) \operatorname{Vol}(A C)}{(s+1)(N(\sigma) s+\nu(\sigma))} \\
+ & \frac{\operatorname{mult}\left(\Delta_{A}\right)}{(N(\tau) s+\nu(\tau))(N(\sigma) s+\nu(\sigma))}+\frac{\operatorname{mult}\left(\Delta_{C}\right)}{\left(N\left(\tau_{1}\right) s+\nu\left(\tau_{1}\right)\right)(N(\sigma) s+\nu(\sigma))} \\
+ & \frac{\operatorname{mult}\left(\Delta_{B 1}\right)}{(N(\tau) s+\nu(\tau))(N(\sigma) s+\nu(\sigma))\left(N\left(\tau_{2}\right) s+\nu\left(\tau_{2}\right)\right)} \\
+ & \frac{\operatorname{mult}\left(\Delta_{B 2}\right)}{\left(N\left(\tau_{2}\right) s+\nu\left(\tau_{2}\right)\right)\left(N\left(\tau_{1}\right) s+\nu\left(\tau_{1}\right)\right)(N(\sigma) s+\nu(\sigma))} .
\end{aligned}
$$

Analogously to Case 1 , we find that

$$
\begin{gathered}
\operatorname{mult}\left(A B^{\circ}\right)=\operatorname{mult}\left(\Delta_{A}\right)=a_{\sigma}^{z}, \operatorname{mult}\left(A C^{\circ}\right)=\operatorname{gcd}\left(a_{\sigma}^{x}, a_{\sigma}^{z}\right)=1=\operatorname{Vol}(A B)=\operatorname{Vol}(B C), \\
\operatorname{Vol}(A C)=2 \operatorname{Vol}(\sigma), \quad \operatorname{mult}\left(B C^{\circ}\right)=\operatorname{mult}\left(\Delta_{C}\right)=e a_{\sigma}^{x}-c a_{\sigma}^{z} .
\end{gathered}
$$

As $\tau$ and $\sigma$ give rise to the same candidate pole (and thus the line through $A$ and $B$ intersects the diagonal of the first quadrant), we also have that $l(m-1)=k(n-1)$. If $m=1$, then also $n=1$ (because $k \geq m$ in this situation) and then $\nu(\tau) / N(\tau)=1$. So now suppose that $m \neq 1$. Then we also use the following relations:

$$
l=\frac{k(n-1)}{(m-1)}, \quad d=c(r-m)+e(t-n), \quad a_{\sigma}^{y}=a_{\sigma}^{x}(k-m)+a_{\sigma}^{z}(l-n), \quad a_{\sigma}^{x}=\frac{a_{\sigma}^{z}(t-l)}{(k-r)}
$$

and we find that $(N(\tau) s+\nu(\tau))(N(\sigma) s+\nu(\sigma))$ disappears when putting everything over the same denominator.

Case 6. $s_{0}$ is contributed by at least three $B_{1}$-facets with respect to the same variable, having line segments in common.

The situation we study is as in Figure 17, where $\sigma_{1}$ and $\sigma_{2}$ might be noncompact. As the facets on the picture all contribute the same candidate pole, the diagonal of the first quadrant must contain the point $(a, b, 1)$. We conclude that this candidate pole is equal to -1 .

Case 7. $s_{0}$ is contributed by various groups of $B_{1}$-facets as in the previous cases, having at most a point in common. 


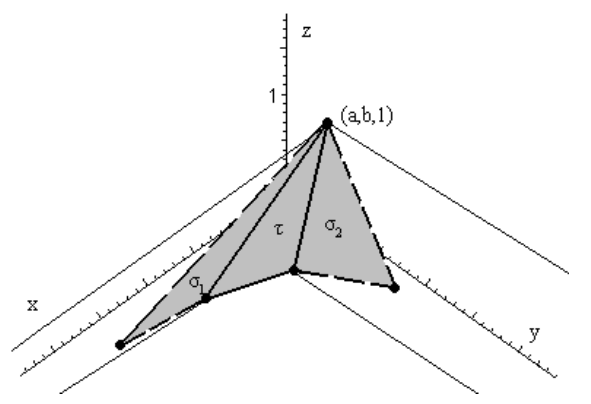

FiguRE 17

In the previous cases we subdivided cones dual to the vertices in such a way that we can split the topological zeta function into parts corresponding to exactly one case. So Case 7 is an immediate consequence of the previous cases.

4.3. The monodromy conjecture for nondegenerate surface singularities. In Theorem 10 we showed that every candidate pole contributed by a facet which is not a $B_{1}$-facet induces an eigenvalue of monodromy, and in Proposition 14 we showed that almost all candidate poles that are only contributed by $B_{1}$-facets are not actual poles. We can now finish our proof of the monodromy conjecture for nondegenerate singularities.

Theorem 15. Let $f$ be a polynomial in three variables which is nondegenerate with respect to its Newton polyhedron $\Gamma_{0}$. If $s_{0}$ is a pole of its local topological zeta function, then $e^{2 \pi i s_{0}}$ is an eigenvalue of monodromy at a point in the neighbourhood of the origin.

Proof. It remains to study candidate poles that are contributed by two facets which are $B_{1}$-facets with respect to different variables and which have a one-dimensional intersection. We may suppose that the two facets are not $B_{1}$-facets with respect to the same variable.

If the two $B_{1}$-facets have a half-line in common, say in the direction of the $z$-axis, then this half-line has equation $x=1, y=1$. The intersection point of this half-line with the diagonal of the first quadrant is the point $(1,1,1)$, and thus the candidate pole is -1 .

If the common line segment is compact, one can check that the only possibility for this configuration is that this segment has vertices $(a, 0,0)$ and $(b, 1,1)$ for $a, b \in \mathbb{Z}_{\geq 0}$. The candidate pole contributed by these facets can be computed by searching the intersection point of the line through $(a, 0,0)$ and $(b, 1,1)$ with the diagonal of the first quadrant. This leads to the value $-\frac{1-b+a}{a}$. The complex number $e^{-2 \pi i(1-b+a) / a}$ is an eigenvalue of monodromy, since the factor $\zeta_{(a, 0,0)}$ does not show up in any $F_{\tau}$ and hence $\left(1-t^{a}\right)$ is a factor of the zeta function of monodromy. (See equation (2).) There might be a cancellation if $e^{-2 \pi i(1-b+a) / a}$ is also a zero of some $\zeta_{\sigma}$ with $\sigma$ a compact line segment which is not contained in any compact facet. However, in this case we can use the argument of the proof of Theorem 10 again to show that $e^{-2 \pi i(1-b+a) / a}$ is an eigenvalue of monodromy at a point in the neighbourhood of the origin. This observation together with Theorem 10 and 
Proposition 14 leads to a proof of the monodromy conjecture for nondegenerate polynomials in three variables.

\section{EXAMPLES}

Example 2. We study the polynomial $f(x, y, z)=x^{12}+y^{13}+z^{14}+x y^{7}+y^{2} z^{4}+$ $x^{2} y^{2} z^{3}$. The picture of the Newton polyhedron in the origin of $f$ is given in Figure 18. The polynomial is nondegenerate and its topological zeta function is

$$
Z_{t o p, f}(s)=\frac{23310 s^{3}+53211 s^{2}+37410 s+8041}{(84 s+43)(s+1)(42 s+17)(27 s+11)} .
$$

We want to illustrate which facets induce eigenvalues of monodromy.

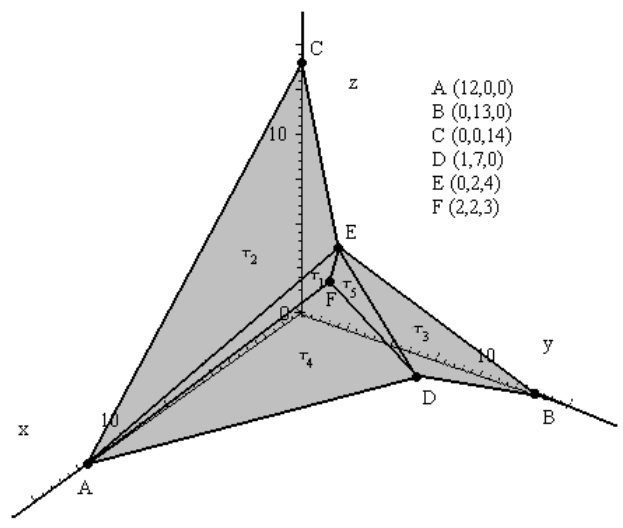

FigURE 18

$\operatorname{Aff}\left(\tau_{1}\right) \leftrightarrow x+2 y+2 z=12$ : This facet does not contain a line segment in a coordinate hyperplane. Hence $F_{\tau_{1}}=\zeta_{\tau_{1}}=\left(1-t^{12}\right)^{2}$. The contributed candidate pole $-5 / 12$ of the topological zeta function is not an actual pole. However, the facet is not a $B_{1}$-simplex and $e^{-10 \pi i / 12}$ is an eigenvalue of monodromy.

$\operatorname{Aff}\left(\tau_{2}\right) \leftrightarrow 7 x+30 y+6 z=84$ : This facet contains two line segments in a coordinate hyperplane. So we include these line segments and their intersection point in $F_{\tau_{2}}$ and find

$$
F_{\tau_{2}}=\frac{\left(1-t^{84}\right)^{4}\left(1-t^{14}\right)}{\left(1-t^{84}\right)^{2}\left(1-t^{14}\right)^{2}}=\frac{\left(1-t^{84}\right)^{2}}{\left(1-t^{14}\right)} .
$$

This is a polynomial having $e^{-86 \pi i / 84}$ as a root.

$\operatorname{Aff}\left(\tau_{3}\right) \leftrightarrow 24 x+4 y+11 z=52$ : Again, the polynomial $F_{\tau_{3}}$ is built with factors arising from the facet, two line segments and a vertex:

$$
F_{\tau_{3}}=\frac{\left(1-t^{52}\right)\left(1-t^{13}\right)}{\left(1-t^{13}\right)\left(1-t^{52}\right)}=1 \text {. }
$$

We see that the candidate pole contributed by $\tau_{3}$ does not induce a zero of this polynomial. Notice that $\tau_{3}$ is a $B_{1}$-facet. Indeed, its vertices are $(0,13,0),(0,2,4)$ and $(1,7,0)$. 
$\operatorname{Aff}\left(\tau_{4}\right) \leftrightarrow 7 x+11 y+16 z=84$ : The facet $\tau_{4}$ contains one line segment in a coordinate hyperplane. Its contribution to the zeta function of monodromy is

$$
F_{\tau_{4}}=\frac{\left(1-t^{84}\right)^{3}}{\left(1-t^{84}\right)}=\left(1-t^{84}\right)^{2} .
$$

The candidate pole $-17 / 42$ (which is a pole) induces a zero of this polynomial.

$\operatorname{Aff}\left(\tau_{5}\right) \leftrightarrow 5 x+7 y+10 z=54$ : The corresponding polynomial of $\tau_{5}$ is equal to $\zeta_{\tau_{5}}$. So

$$
F_{\tau_{5}}=1-t^{54} \text {. }
$$

The pole $-11 / 27$ is contributed by $\tau_{5}$ and $e^{-22 \pi i / 27}$ is a zero of $F_{\tau_{5}}$.

The only vertex on a coordinate axis that belongs to more than one facet is the point $(12,0,0)$. To write the monodromy zeta function of $f$ in the origin, we take the product of $\left(1-t^{12}\right)$ with the factors $F_{\tau_{i}}$. We conclude that

$$
Z(f, 0)(t)=\frac{\left(1-t^{12}\right)^{3}\left(1-t^{84}\right)^{4}\left(1-t^{54}\right)}{1-t^{14}}
$$

One can verify that the conditions for Theorem 3 are not satisfied for $\tau=\tau_{4}$. Indeed, take $\sigma=\tau_{2}$. Then $\beta(\tau, \sigma)$ is the greatest common divisor of the $(2 \times 2)$ minors of the matrix

$$
\left(\begin{array}{ccc}
7 & 11 & 16 \\
7 & 30 & 6
\end{array}\right)
$$

which is equal to 1 , and $\lambda(\tau, \sigma)=43-\frac{34}{84} 84=9$. Hence, $\mu(\tau, \sigma)$ is an integer.

The next example shows the situation when the Newton polyhedron contains a line segment in a coordinate plane which is not contained in a compact facet.

Example 3. We study the polynomial $f(x, y, z)=x^{9} y+x^{2} y^{3}+x y^{6} z^{2}+y^{5} z^{5}$. Its Newton polyhedron at the origin is pictured in Figure 19. It has 4 vertices:

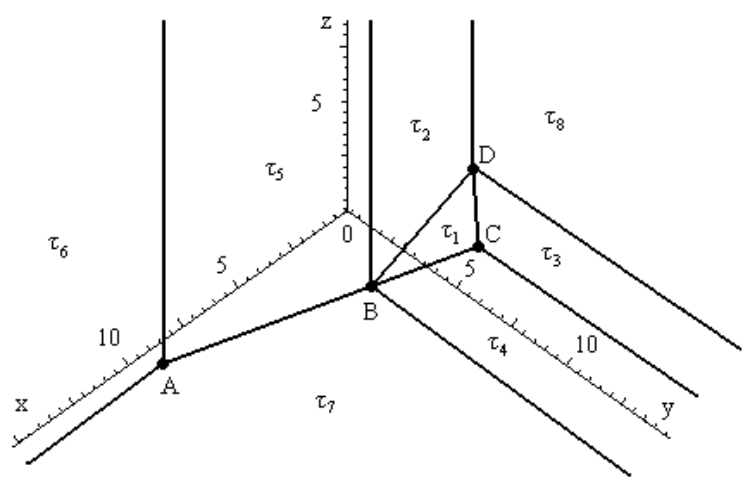

FIGURE 19

$A(9,1,0), B(2,3,0), C(1,6,2)$ and $D(0,5,5)$. The 8 facets have the following equations:

$$
\begin{gathered}
\operatorname{Aff}\left(\tau_{1}\right) \leftrightarrow 11 x+y+4 z=25, \quad \operatorname{Aff}\left(\tau_{2}\right) \leftrightarrow x+y=5, \\
\operatorname{Aff}\left(\tau_{3}\right) \leftrightarrow 3 x+z=5, \quad \operatorname{Aff}\left(\tau_{4}\right) \leftrightarrow 2 x+z=4, \quad \operatorname{Aff}\left(\tau_{5}\right) \leftrightarrow 2 x+7 y=25, \\
\operatorname{Aff}\left(\tau_{6}\right) \leftrightarrow y=1, \quad \operatorname{Aff}\left(\tau_{7}\right) \leftrightarrow z=0, \quad \operatorname{Aff}\left(\tau_{8}\right) \leftrightarrow x=0 .
\end{gathered}
$$


Its local topological zeta function is

$$
Z_{t o p, f}(s)=\frac{2685 s^{3}+6026 s^{2}+4110 s+864}{(5 s+2)(4 s+3)(25 s+9)(s+1)(25 s+16)} .
$$

The zeta function of monodromy at the origin is equal to $F_{\tau_{1}}\left(=\zeta_{\tau_{1}}\right)$ divided by $\zeta_{\sigma}$, where $\sigma$ is the compact line segment $A B$. Both factors are equal to $\left(1-t^{25}\right)$, so the zeta function of monodromy at the origin is equal to 1 . The facet $\tau_{1}$ is no $B_{1}$-facet. It induces the pole $-16 / 25$ of the local topological zeta function. As in the proof of Theorem 10 we need to consider a complex number $k$ such that $f(x, y, z-k)$ is nondegenerate with respect to its Newton polyhedron at the origin. This Newton polyhedron $\Gamma_{k}$ is as in Figure 20. We have vertices $(9,1,0),(2,3,0)$ and $(0,5,0)$.

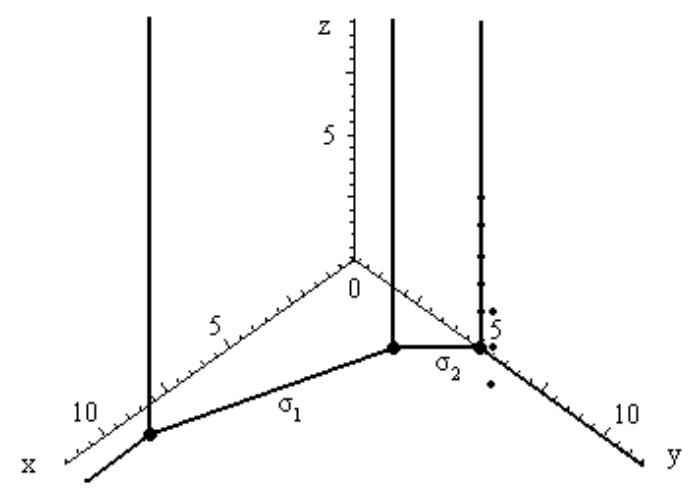

Figure 20

The zeta function of monodromy of $f$ at the point $(0,0, k)$ is equal to

$$
\left(\zeta_{\sigma_{1}}\right)^{-1}\left(\zeta_{\sigma_{2}}\right)^{-1} \zeta_{(0,5,0)}=\frac{\left(1-t^{5}\right)}{\left(1-t^{5}\right)^{2}\left(1-t^{25}\right)}
$$

We conclude that $e^{-32 \pi i / 25}$ is an eigenvalue of monodromy at a point in the neighbourhood of the origin. Moreover, note that the candidate pole $-4 / 5$, contributed by $\tau_{3}$, is not a pole. Indeed, this could be predicted since the facet $\tau_{3}$ is a $B_{1}$-facet with respect to the variable $x$.

\section{REFERENCES}

[A'C75] N. A'Campo, La fonction zêta d'une monodromie, Comment. Math. Helv. 50 (1975), 233-248. MR0371889 (51:8106)

[AVG86] V. Arnold, A. Varchenko and S. Goussein-Zadé, Singularités des applications différentiables II, Editions Mir, Moscou (1986).

[A-B,C-N] E. Artal Bartolo, P. Cassou-Noguès, I. Luengo and A. Melle Hernández, Monodromy conjecture for some surface singularities, Ann. Scient. Ecole Norm. Sup. 35 (2002), 605-640. MR1981174 (2004e:32030)

[A-B,C-N1] E. Artal Bartolo, P. Cassou-Noguès, I. Luengo and A. Melle Hernández, Quasiordinary power series and their zeta functions, Memoirs Amer. Math. Soc. 178, no. 841 (2005). MR2172403 (2007d:14005)

[At70] M.F. Atiyah, Resolution of singularities and division of distributions, Comm. Pure Appl. Math. 23 (1970), 145-150. MR 0256156 (41:815)

[BeGe69] I.N. Bernstein and S.I. Gel'fand, Meromorphic property of the function $P^{\lambda}$, Funct. Anal. Appl. 3 (1969), 68-69. MR0247457(40:723) 
[D95] J. Denef, Poles of p-adic Complex Powers and Newton Polyhedra, Nieuw Archief voor Wiskunde (4), 13 (1995), 289-295. MR1378800 (96m:11106)

[DL92] J. Denef and F. Loeser, Caractéristique d'Euler-Poincaré, fonctions zêta locales et modifications analytiques, J. Amer. Math. Soc. 5, 4 (1992), 705-720. MR.1151541 (93g:11118)

[Ig75] J. Igusa, Complex powers and asymptotic expansions I, J. reine angew. Math. 268/269 (1974), 110-130; II, ibid 278/279 (1975), 307-321. MR0347753 (50:254) MR0404215 (53:8018)

[Ig78] J. Igusa, Lectures on forms of higher degree, Tata Inst. Fund. Research, Bombay (1978). MR 546292 (80m:10020)

[Ig88] J. Igusa, B-functions and p-adic integrals, Algebraic Analysis, Academic Press (1988), 231-241. MR992457 (90i:11140)

[LVa09] A. Lemahieu and L. Van Proeyen, Poles of the topological zeta function for plane curves and Newton polyhedra, C. R. Acad. Sci. 347 (2009), 637-642. MR2532921

[LV09] A. Lemahieu and W. Veys, Zeta functions and monodromy for surfaces that are general for a toric idealistic cluster, International Mathematics Research Notices, No. 1 (2009) 1162. MR2471295

[L88] F. Loeser, Fonctions d'Igusa p-adiques et polynômes de Bernstein. Amer. J. Math. 110 (1988), 1-22. MR926736 (89d:11110)

[L90] F. Loeser, Fonctions d'Igusa p-adiques, polynômes de Bernstein, et polyèdres de Newton, J. reine angew. Math. 412 (1990), 75-96. MR1079002 (92c:11139)

[Ma83] B. Malgrange, Polynômes de Bernstein-Sato et cohomologie évanescente, Astérisque 101/102 (1983), 243-267. MR737934 (86f:58148)

[Mi68] J. Milnor, Singular points of complex hypersurfaces, Ann. of Math. Studies Vol. 61, Princeton University Press (1968). MR0239612(39:969)

[Ro04] B. Rodrigues, On the monodromy conjecture for curves on normal surfaces, Math. Proc. of the Cambridge Philosophical Society 136 (2004), 313-324. MR2040577 (2005g:14006)

[Var76] A.N. Varchenko, Zeta-Function of Monodromy and Newton's Diagram, Inventiones Math. 37 (1976), 253-262. MR0424806 (54:12764)

[Ve95] W. Veys, Determination of the poles of the topological zeta function for curves, Manuscr. Math. 87 (1995), 435-448. MR1344599(97a:11192)

[We65] A. Weil, Sur la formule de Siegel dans la théorie des groupes classiques, Acta Math. 113 (1965), 1-87. MR0223373(36:6421)

UFR de Mathématiques, Université Lille 1, 59655 Villeneuve d’AscQ, France

E-mail address: lemahieu.ann@gmail.com

Departement Wiskunde, K. U. Leuven, Celestijnenlaan 200B, B-3001 Leuven, Belgium

E-mail address: lisevanproeyen@gmail.com 